\title{
Europäische Sozialpolitik und Europas parteipolitisches Gravitationszentrum in den Jahren 1957-2003
}

Regierungen vertreten in internationalen Verhandlungen nationale Interessen. Welchen Einfluss übt die ideologische Färbung einer Regierung auf ihre Verhandlungspositionen aus? Gelingt eine Einigung leichter, wenn es eine hohe parteipolitische Übereinstimmung in der Zusammensetzung der Regierungen gibt? In diesem Aufsatz zeigen wir, weshalb die Analyse der Parteipolitik auch in der internationalen Politik mehr Beachtung finden sollte. Wir betrachten das parteipolitische Gravitationszentrum der Europäischen Union und liefern eine erste systematische Darstellung der Regierungszusammensetzung aller Mitgliedsstaaten von 1957 bis 2003. Darüber hinaus wird untersucht, wie integrationsfreundlich, links oder rechts orientiert sowie ideologisch homogen bzw. heterogen die Mitgliedsstaaten - und damit intergouvernementale EU-Gremien - im Zeitverlauf waren. Hierbei greifen wir auf Expertensurveys, Daten zur parteipolitischen Regierungszusammensetzung und Daten des Manifesto-Projekts zurück. Eine Fallstudie zur Entwicklung der EU-Sozialpolitik seit den Römischen Verträgen ergänzt die quantitative Darstellung. Sie unterstreicht, dass die Analyse internationaler Verhandlungen ein angemessenes Verständnis der parteipolitischen Dimension des Einigungsprozesses voraussetzt.

\section{Einleitung: Parteien und nationale Interessen ${ }^{1}$}

Die spanischen Parlamentswahlen im März 2004 veränderten mit dem Regierungswechsel Spaniens Außenpolitik. Wie im Wahlkampf angekündigt, zog der neue sozialistische Regierungschef José Luis Rodríguez Zapatero die Truppen seines Landes aus dem Irak zurück. Der Übergang von den Demokraten zu den Republikanern beeinflusste nicht nur die strategische Ausrichtung der amerikanischen Außenpolitik, sondern machte auch eine Einigung im Streit um das Kyoto-Protokoll unmöglich. Die Beschäftigungsstrategie der EU konnte erst verabschiedet werden, nachdem in Großbritannien die Labour Party die Konservativen abgelöst hatte. Die parteipolitische Ausrichtung nationaler Regierungen schlägt sich in der Interpretation nationaler Interessen nieder und übt einen Einfluss auf die Einigungschancen in internationalen Verhandlungen aus. Regierungsparteien »verkoppeln« die nationale und internationale Ebene. Sie werden in internationalen Verhandlungen keine Posi-

1 Wir danken Christian Joerges, Stephan Leibfried, Miriam Hartlapp, Ulrich Sedelmeier, Wolfgang Streeck, Oliver Treib und drei anonymen Gutachterinnen bzw. Gutachtern der ZIB für zahlreiche Hinweise. Die verbleibenden Unzulänglichkeiten sind ausschließlich uns zuzuschreiben. 
tionen einnehmen, die ihrer nationalen politischen Programmatik eindeutig widersprechen (Marks/Wilson/Ray 2002: 586). ${ }^{2}$

In diesem Aufsatz untersuchen wir einen Faktor, der die Kooperationswahrscheinlichkeit zwischen Staaten beeinflusst, aber von der Theorie der Internationalen Beziehungen nach unserem Eindruck bislang kaum beachtet worden ist: die parteipolitische Zusammensetzung der Regierung. Wir untersuchen den Einfluss der parteipolitischen Ausrichtung auf die zwischenstaatliche Kooperation an einem Fall, für den ein solcher Einfluss am ehesten erwartet werden kann: für die EU. Sie hat sich am weitesten von einem internationalen Regime zu einem eigenständigen politischen System entwickelt - u. a. begünstigt durch die homogene parteipolitische Zusammensetzung der Regierungen in ihrer Gründungsphase! -, und daher ist ein Einfluss der parteipolitischen Zusammensetzung der EU-Mitgliedsregierungen auf die Europapolitik in vielen Studien immer wieder vermerkt worden, ohne dass dies bislang Anlass zu einer systematischen Untersuchung von Europas parteipolitischem Gravitationszentrum gegeben hätte. ${ }^{3}$ Wir fragen im Folgenden, a) wie sich die Regierungszusammensetzung in den EU-Mitgliedsländern auf die parteipolitische Zusammensetzung des Europäischen Rats und des Ministerrats der EU ausgewirkt hat und b) welche Politikkonsequenzen unterschiedliche Mehrheiten im Rat für die europäische Sozialpolitik besessen haben. Unsere allgemeine, über die Europäische Union hinausweisende Ausgangshypothese ist einfach: Je ähnlicher die parteipolitischen Positionen der Staaten sind, für desto wahrscheinlicher halten wir ceteris paribus zwischenstaatliche Kooperation, und je eindeutiger die parteipolitischen Mehrheitsverhältnisse in zwischenstaatlichen Entscheidungsgremien ausfallen, desto deutlicher sollte sich dies auf den Inhalt dieser Kooperation auswirken. Damit wird zugleich die Grenze der hier vorgeschlagenen Herangehensweise deutlich. In internationalen Regimen mit großer Mitgliederzahl ist die Wahrscheinlichkeit ausgeprägter Links-rechts-Schwankungen geringer als beispielsweise in der früheren Europäischen Gemeinschaft. Die Bedeutung der Parteipolitik für die nationale Verhandlungsposition wird zudem größer sein, wenn internationale Entscheidungen innenpolitisch stark umstrittene Politikfelder betreffen.

Unsere Untersuchung ist ein erster Schritt in der Analyse der parteipolitischen Beeinflussung zwischenstaatlicher Verhandlungsregime. Was den exemplarischen Untersuchungsgegenstand dieser Studie anbetrifft, die Europäische Union, so wollen wir mit der Betonung der parteipolitischen Dimension der europäischen Einigung keine eigenständige Integrationstheorie formulieren, sondern bestehende

2 Allerdings gibt es keinen Grund für die Annahme, dass die Bedeutung der parteipolitischen Ausrichtung einer Regierung in allen Politikfeldern gleich groß ist. In welchen Fällen politische Ideologie die nationalen Präferenzen beeinflusst, bleibt eine empirische Frage. Generell beurteilen wir Andrew Moravcsiks (1997) Argumentation skeptisch, dass sich Regierungen in internationalen Verhandlungen von innenpolitischen Ansprüchen emanzipieren können. Der nationale Parteienwettbewerb drängt Parteien, ein kohärentes ideologisches Profil zu bewahren (Hinich/Munger 1992).

3 Allerdings hat Martin Höpner (2005) in der letzten Ausgabe der Zeitschrift für Internationale Beziehungen auf die Bedeutung parteipolitischer Faktoren für die europäische Finanzmarktintegration hingewiesen. 
Erklärungsansätze ergänzen. So stimmen verschiedene Ansätze in den Internationalen Beziehungen auf ganz allgemeiner Ebene darin überein, dass zwischenstaatliche Kooperation dann stattfindet, wenn sie für die Beteiligten Wohlfahrtsgewinne verspricht (vgl. Keohane 1984; Zürn 1992). Doch was jeweils als im »nationalen Interesse « liegend definiert wird, bemisst sich nicht zuletzt nach den ideologischen bzw. programmatischen Überzeugungen derer, die dieses nationale Interesse in internationalen Verhandlungen vertreten - den nationalen Regierungen. Zum Beispiel werden sozialdemokratische Regierungen die Einigung auf europaweite Sozialstandards eher als wohlfahrtssteigernd wahrnehmen als konservative Regierungen; Letztere dürften eher geneigt sein, die Durchsetzung der vier Wirtschaftsfreiheiten als nationalen Wohlfahrtsgewinn zu verstehen. Die Wahrscheinlichkeit der Einigung auf europäische Re-Regulierung oder Deregulierung bestimmt sich daher auch nach der parteipolitischen Ausrichtung der EU-Mitgliedsländer, die sich im Rat auf eine gemeinsame Politik einigen. Entsprechend ist es plausibel zu erwarten, dass linke Regierungen in internationalen Verhandlungen eher protektionistische Positionen vertreten, während rechte Regierungen stärker für wirtschaftliche Deregulierung eintreten. Die Bedeutung der programmatischen Positionen von Staaten weist daher über den spezifischen EU-Kontext hinaus.

Wir untersuchen im Folgenden systematisch die parteipolitische Zusammensetzung der EU-Mitgliedsregierungen seit 1957. Damit können wir eine Positionierung des Europäischen Rats und des Ministerrats anhand von zwei Dimension vornehmen: der grundlegenden Links-rechts-Dimension, wie auch der »Integration vs. nationale Souveränität«-Dimension (Hix/Lord 1997: 50). Verbunden ist unsere Analyse mit einer Fallstudie zur europäischen Sozialpolitik von den Römischen Verträgen bis zum Amsterdamer Vertrag. Die Fallstudie dient zum einen als plausibility probe, d. h. sie bietet Evidenz dafür, dass Europas sozialpolitischer Integrationspfad auch von der parteipolitischen Ausrichtung der EU-Mitgliedsregierungen beeinflusst worden ist. Die Fallstudie hat zum anderen die Funktion, einige Aspekte der parteipolitischen Dimension des EU-Einigungsprozesses zu beleuchten, die mit einer rein quantitativen Betrachtung schwer zu erfassen sind. In dieser Hinsicht ist insbesondere die Berücksichtigung innerparteilicher Strömungen und Flügel bei der Kompromissbildung über den europäischen Integrationspfad von Bedeutung. Die Fallstudie bietet somit nicht nur einen Plausibilitätsnachweis, sondern auch eine wichtige Ergänzung zur quantitativ-empirischen Untersuchung.

Der Aufsatz ist wie folgt aufgebaut: Zunächst begründen wir in Abschnitt 2, weshalb und in welcher Hinsicht wir von der parteipolitischen Zusammensetzung einer Regierung einen Einfluss auf ihre Haltung in zwischenstaatlichen Verhandlungen und damit auch auf das Ergebnis dieser Verhandlungen selbst erwarten können. Abschnitt 3 erläutert unser methodisches Vorgehen und bietet dann einen systematisch-quantitativen Überblick über Europas parteipolitisches Gravitationszentrum von 1957 bis zum Jahr 2003; insbesondere verorten wir den Ministerrat auf der Links-rechts- und der Integrations-Skala. In beiden Dimensionen können die Regierungen einheitliche oder divergierende Positionen einnehmen. Das heißt, nicht nur die Lage des Gravitationszentrums zu einem gegebenen Zeitpunkt, sondern auch die 
Homogenität der Positionen geben Auskunft darüber, ob und in welchen Feldern Kooperation wahrscheinlich ist. Indem wir »horizontale« und »vertikale « Perspektiven zusammenbringen, wird deutlich, dass Regierungsparteien aus unterschiedlichen Gründen für oder gegen die Ausweitung der EU-Kompetenzen sein können. Dies zeigt Abschnitt 4, in dem wir die Geschichte der EU Sozialpolitik von ihren Anfängen bis heute unter dem besonderen Blickwinkel ihrer parteipolitischen Dimension rekonstruieren. Ein letzter Abschnitt enthält einen Ausblick auf weitere Forschungsfragen, die sich aus unseren Befunden ergeben.

\section{Parteipolitische Programmatik, nationale Interessen und die Wahrscheinlichkeit internationaler Kooperation - die EU als Anwendungsfall}

Es gibt in der EU-Literatur anekdotische Evidenz, die auf die Bedeutung der parteipolitischen Mehrheitsverhältnisse unter den EU-Mitgliedsländern für die europäische Integration verweist. Hierzu gehören z. B. die Hinweise auf den Einfluss christdemokratischer Hegemonie unter den sechs Gründungsstaaten in den 1950er Jahren für die frühe Konstitutionalisierungsphase der EU, die Bedeutung des parteiübergreifenden Charakters der deutsch-französischen Zusammenarbeit in den 1970er, 1980er und 1990er Jahren oder die Auswirkung des »Linksrucks « unter den EUMitgliedsstaaten in der zweiten Hälfte der 1990er Jahre für sozialpolitische Initiativen, wie z. B. das Beschäftigungskapitel des Amsterdamer Vertrags. Doch die vielen Einzelverweise haben bislang noch zu keiner systematischen Untersuchung von Europas parteipolitischer Dimension geführt. Was sind vermutliche Gründe für diese Aussparung?

In der Forschung zur europäischen Integration dominierte lange Zeit eine »vertikale « Perspektive, d. h. es stand die Frage nach dem »Mehr« oder »Weniger « an Integration im Mittelpunkt, während die »horizontale« Links-rechts-Dimension des europäischen Integrationsprojekts demgegenüber deutlich in den Hintergrund trat (Genschel 1998). Integration wurde vornehmlich als Delegation nationalstaatlicher Souveränitätsrechte oder als funktionaler spillover von Kompetenzen auf die supranationale Ebene verstanden. Hierin stimmten die großen Integrationstheorien wie Realismus, Intergouvernementalismus und Funktionalismus überein. Ihnen gemeinsam war die Dichotomisierung von nationaler und internationaler Politik, die die parteipolitische Dimension zwischenstaatlicher Kooperation vernachlässigte. ${ }^{4}$ Dem entspricht eine analytische Perspektive bei der Darstellung und Untersuchung der einzelnen EU-Institutionen und Akteure, die diese entweder als bürokratische oder

4 Der »liberale Intergouvernementalismus« (Moravcsik 1991, 1993, 1998) verbindet nationale Politik und internationale Verhandlungen. Allerdings strebt er eine politökonomische, sektorale Erklärung nationaler Verhandlungspositionen an, bei der vor allem nationale Produzenteninteressen im Vordergrund stehen (Moravcsik 1998: 33-38). Die Dichotomisierung von nationaler und internationaler Politik wurde in der EU-Forschung auch durch den Rückgriff auf das Konzept des »Zwei-Ebenen-Spiels« (Putnam 1988) aufgelöst. Am Beispiel der Währungsunion haben dies John Woolley (1994) sowie Dieter Wolf und Bernhard Zangl (1996) durchexerziert. 
als intergouvernementalistische Gebilde versteht, ihnen jedoch eine genuin parteipolitische Prägung abspricht. Während der Ministerrat vornehmlich als Ort nationaler Interessenvertretung analysiert wird, begreift die Literatur die Kommission als »Hüterin der Verträge« sowie als Bürokratie mit einem Eigeninteresse an Kompetenzausweitung (Sandholtz/Stone Sweet 1998; Stone Sweet et al. 2002; Pollack 1997, 2003). Als weiterer wichtiger, aber wiederum eher »unpolitischer « Akteur wird der Europäische Gerichtshof (EuGH) in den Blick genommen (Alter 1998; Alter/Meunier-Aitsahalia 1994; Burley/Mattli 1993; Stone Sweet 2004). Kommission und EuGH besitzen beinahe per definitionem, auf alle Fälle per Mission, einen überparteilichen Auftrag zur Förderung der europäischen Integration, sodass ihre politikwissenschaftliche Betrachtung oft neofunktionalistisch eingefärbt ist, während bei der Betrachtung des Ministerrats eher die Semantik des Intergouvernementalismus vorherrscht. Dass und in welchem Ausmaß diese Interessen auch jeweils parteipolitisch bestimmt sind, wurde hingegen bislang nicht detailliert erörtert, obwohl es zunehmend Hinweise auf die Bedeutung der parteipolitischen Dimension für die Entscheidungsfindung im Rat gibt (Aspinwall 2002; Mattila 2004).

Ähnliche Aussparungen lassen sich für die Literatur über Europas demokratische Dimension feststellen. Wenn in der Europaforschung Wahlen analysiert werden, wurde bislang fast ausschließlich die Rolle der Europawahlen für die Zusammensetzung des Parlaments und das Verhältnis zwischen nationalen und Europawahlen untersucht (Reif/Schmitt 1980; Reif 1984; van der Eijk et al. 1996; van der Eijk/ Franklin 1996), nicht aber die Bedeutung nationaler Wahlen für die Zusammensetzung von Ministerrat und Europäischem Rat und für das Zusammenspiel zwischen Rat, Kommission und Parlament. Alternativ interessierte die Auswirkung der europäischen Integration auf die nationalen Parteiensysteme (Mair 2001; Bartolini 2005: Kap. 6), nicht aber die Bedeutung der domestic politics für den europäischen Integrationsprozess. Zugleich wird aber auf die Bedeutung gerade dieses Zusammenhanges immer wieder hingewiesen. So zählt etwa Simon Hix in seiner umfassenden Behandlung des »Politischen Systems der EU« nationale Wahlen zu den »most important channels through which EU-citizens can exert influence and can make demands on the EU system: In national elections citizens choose governments who subsequently represent these citizens in the Council« (Hix, 1999: 5). Aber welche Parteien haben Europas Wähler mit ihrer Repräsentation »in Brüssel« betraut? Hix und Christopher Lord heben zudem hervor, dass europapolitische Integrationsschübe insbesondere dann zu verzeichnen sind, wenn Regierungen »günstige Situationen« (»favorable conjunctures«; Hix/Lord 1997: 4) ausnutzen wollen, um ihre Nachfolgeregierungen politisch zu binden. Was aber eine europapolitisch günstige Situation ist, bemisst sich erneut auch an der parteipolitischen Zusammensetzung des Ministerrats, wenn nicht sogar primär daran.

Wie sich leicht zeigen lässt, besitzt die Betrachtung der parteipolitischen Zusammensetzung der EU-Mitgliedsregierungen für eine Reihe von theoretischen Argumenten der Europaliteratur zentrale Bedeutung. So argumentiert etwa George Tsebelis, dass das Europäische Parlament dann zum »konditionalen Agenda-Setzer « werden kann, wenn im Ministerrat heterogene Positionen einer einheitlichen Wil- 
lensbildung entgegenstehen (Tsebelis 1994: 135-136; Tsebelis/Kreppel 1998). Die Interessenheterogenität im Ministerrat ist aber unzweifelhaft oft auch parteipolitischer Natur. Analog bestimmt sich auch das Delegationsverhältnis zur Kommission nach dem Grad der Interesseneinigkeit im Rat (Pollack 2003: 34). Dem Problem der »bürokratischen Drift«, also der aktivistischen Selbstbeauftragung der Kommission in wichtigen Integrationsfragen, kann mit institutionellen Checks begegnet werden. Diese Delegationsproblematik würde sich allerdings nur begrenzt stellen, wenn der Ministerrat in zentralen Sachfragen eine einheitliche Position bezöge. Es ist plausibel anzunehmen, dass den Mitgliedsländern eine Einigung im Rat dann leichter fällt, wenn sie eine gleiche parteipolitische Ausrichtung haben. Wie man sieht, schlägt hier die Heterogenität bzw. Homogenität der politischen Positionen der EU-Mitgliedsregierungen unmittelbar auf die europäische Verfassungspraxis, d. h. auf die Machtgewichte zwischen Rat, Parlament und Kommission, durch.

Die Bedeutung von Europas parteipolitischer Dimension verdeutlicht auch Paul Piersons (1996) Argument, dass aufgrund des »Präferenzwandels « nationaler Regierungen die EU zu keinem Zeitpunkt eine direkte Entsprechung der Wünsche der Einzelstaaten sein kann, wie es der Intergouvernementalismus oder auch der Realismus nahe legen. Eine der wichtigsten Quellen von Präferenzwandel sind - laut Pierson (1996: 140) - Regierungswechsel. Die Delegation von Entscheidungsbefugnissen an suprastaatliche Instanzen wird auch oft als eine Strategie interpretiert, mit der sich Staaten wechselseitig glaubwürdig binden wollen. Doch stellt sich dieses Problem der Selbstbindung insbesondere dann, wenn Regierungen nicht nur das jeweils - relativ stabile - geopolitische oder ökonomische Nationalinteresse vertreten. Regierungen binden sich mit internationalen Vereinbarungen, weil sie sich selbst und die Verhandlungspartner, aber auch ihre Nachfolger disziplinieren wollen. Internationale Kooperation basiert gerade darauf, dass internationale Organisationen ihre Ziele zumindest teilweise unabhängig von den wechselnden Präferenzen der Mitgliedsstaaten verfolgen können. Die Hauptursache für das, was hier abstrakt mit »wechselnden Präferenzen « bezeichnet wird, sind aber durch demokratische Wahlen veranlasste Änderungen in der parteipolitischen Ausrichtung von Regierungen. Die EU-Literatur der letzten Jahre sieht ein Hauptmotiv für den Kompetenztransfer auf die Europäische Kommission und den Gerichtshof in der höheren Glaubwürdigkeit dieser Arrangements (Moravcsik 1998: 67-68; Pollack 1997). Doch es ist weniger der unterstellte Opportunismus der Vertragsparteien, vor dem solche Arrangements schützen sollen, als die durch Regierungswechsel verursachte »zeitliche Inkonsistenz« im Handeln der Staaten. Dies ist keine EU-spezifische Problematik, sondern gilt generell für die wechselseitige Selbstbindung von Staaten in den internationalen Beziehungen. Auch die These, dass viele vertragliche Regelungen in der EU als »institutionalized dispute resolution« zu verstehen sind (Stone Sweet/Sandholtz 1998: 16-17), führt unmittelbar zur Frage nach der jeweiligen parteipolitischen Zusammensetzung der EU-Mitgliedsregierungen, sind doch viele Dispute über die europäische Integration parteipolitisch eingefärbt.

Wir meinen, dass eine Analyse der großen europäischen Konstitutionalisierungsschritte, aber auch die Untersuchung des Machtgleichgewichts zwischen Rat, 
Kommission und Parlament mit ihrer zentralen Bedeutung für Europas gelebte Verfassung, nicht gut ohne eine systematische Betrachtung der parteipolitischen Regierungszusammensetzung in den Mitgliedsländern möglich ist. Gleiches könnte für die Umsetzung von EU-Politiken behauptet werden, wie sich am Stabilitäts- und Wachstumspakt demonstrieren lässt. Zunächst maßgeblich von einer konservativen deutschen Regierung ausgehandelt, die die weniger monetaristischen Länder der Währungsunion auf ein hohes Ausmaß wirtschafts- und währungspolitischer Disziplin verpflichten wollte, führte der Regierungswechsel in Deutschland 1998 dazu, dass das Interesse an einer strikten Interpretation des Maastricht-Regelwerks deutlich abnahm. Neuere Forschungen zur Implementation europäischer Richtlinien streichen ebenfalls die Bedeutung der parteipolitischen Konstellation auf nationaler Ebene heraus (Treib 2003). Auch diese Literatur betont also den Wert, den eine systematischere Langzeitanalyse von Europas parteipolitischem Gravitationszentrum als Ergänzung zur bisherigen Integrationsforschung besitzen würde. Ihr wenden wir uns im folgenden Abschnitt zu.

\section{Wie hat sich die parteipolitische Regierungszusammensetzung in den EU-Mitgliedsländern seit 1955 entwickelt?}

Will man eine Regierung in einem gedachten politischen Raum verorten, bieten sich verschiedene Vorgehensweisen an. Dabei lassen sich zwei Arten von Studien unterscheiden. Zum einen kann man Parteien nach Parteifamilien bzw. auf einer Linksrechts-Skala verorten (siehe für einen Überblick Schmidt 1996). Zum anderen kann man einen ideologischen Gravitationspunkt errechnen (Gross/Sigelman 1984). Diese Verortung ergibt sich aus der Information über die Positionierung einer Partei auf einer Ideologie-Skala und dem Gewicht, mit der diese Position in die Gesamtposition der Regierung eingeht - in Koalitionsregierungen gängigerweise dem jeweiligen Parteianteil an den Abgeordnetensitzen aller Regierungsparteien (bei einer Einparteienregierung ist dieses Gewicht 1). Um die ideologische Positionierung der Parteien zu eruieren, können Expertenbefragungen durchgeführt werden (Laver/Hunt 1992; Castles/Mair 1984; Huber/Inglehart 1995). Sie haben jedoch die bekannten Probleme der Reliabilität und Validität. Zudem bilden Befragungen im Regelfall nur die Situation zu einem gegebenen Zeitpunkt ab. Selbst wenn wir davon ausgehen, dass sich Parteipositionierungen über Zeit nur sehr wenig ändern, stellt das Problem fehlender Werte für zum Zeitpunkt der Expertenbefragung nicht mehr existente Parteien ein ernsthaftes Problem dar, wenn man die Entwicklung über längere Zeiträume verfolgen will.

Eine alternative Vorgehensweise ist die Bestimmung parteipolitischer Positionierungen durch die Inhaltsanalyse von Wahlprogrammen. Diesen Weg hat das groß angelegte Comparative Manifesto Project (CMP, siehe Budge et al. 2001) gewählt, das die Programme für alle Parteien in 25 westlichen Demokratien zwischen 1945 und 1998 auswertet. Gegenüber dem beeindruckenden Ergebnis des CMP-Forschungsverbundes mag man sowohl methodische als auch theoretische Einwände formulieren. So bleibt es fraglich, ob Wahlprogramme tatsächlich die »wirklichen« 
Positionierungen von Parteien repräsentieren und ob und wie sich aus der Frequenz von Nennungen einiger zentraler Schlüsselbegriffe inhaltliche Positionsbestimmungen ableiten lassen. Aus zwei Gründen werden wir im Folgenden trotz aller Einzelkritik sowohl auf die Experten-surveys als auch auf die Daten des Manifesto-Projekts zurückgreifen. Erstens gibt es schlicht keine empirische Alternative. Zweitens bieten die Daten trotz der erwähnten Einwände insbesondere dann wertvolle Informationen über Europas parteipolitisches Gravitationszentrum, wenn man sie stärker in Hinblick auf Veränderungen und Trends über Zeit befragt, anstatt Aufschluss über konkrete Positionierungen einzelner Parteien zu einem gegebenen Zeitpunkt zu erwarten.

Es mögen auch Zweifel hinsichtlich unseres Ansatzes im Kontext der Europäischen Union bestehen: Wenn alle wichtigen EU-Entscheidungen entweder formell oder informell dem Einstimmigkeitsprinzip folgen, was für einen Sinn macht es dann, eine durchschnittliche Position der EU-Mitgliedsregierungen zu errechnen? Wäre es in diesem Fall nicht angemessen, die Position »pivotaler Länder« zu ermitteln? Dies hieße aber in unseren Augen, den zweiten vor dem ersten Schritt zu machen. Die Bestimmung eines Präferenzaußenseiters in einer bestimmten Entscheidungsfrage setzt voraus, dass wir wissen, wo - statistisch gesprochen - der Mittelwert der Grundgesamtheit liegt. Als Annäherung an die Frage, wie weit die Positionen zwischen den Mitgliedsregierungen streuen, berichten wir über die Entwicklung der Standardabweichung unserer Gravitationszentrum-Variablen (siehe Abb. 2). Vor dem Hintergrund dieses Bilds können zukünftige Analysen Extrempositionierungen in ausgewählten issue-Bereichen betrachten, wobei - wie gesagt solche Analysen sich schnell mit dem Problem einer nur begrenzten Datenqualität konfrontiert sehen. Schließlich halten wir auch deswegen die Ermittlung von Durchschnittswerten für sinnvoll, weil das Einstimmigkeitsprinzip in der EU keinem Mitgliedsland für jede Entscheidungsfrage eine vollständige Vetomacht zuweist. Präferenzaußenseiter werden durch die jeweilige Mehrheit diszipliniert und durch etablierte Reziprozitätsregeln an der freien Ausübung ihres formellen Vetorechts gehindert (Héritier 1996; Caporaso 1992; Manow 1999). Selbst Margaret Thatcher konnte in der EU keine Politik des »permanenten Neins « verfolgen.

Wir nutzen für die folgende Analyse also drei Datenquellen: Die von Michael Laver und Ben Hunt (1992) durchgeführte Expertenbefragung zur ideologischen Positionierung von Parteien, die Angaben von Leonard Ray (1999) über die Integrationsorientierung bzw. -skepsis von Parteien in den EU-Mitgliedsländern und schließlich die Daten des Comparative Manifesto Project (Budge et al. 2001). Zunächst aber gruppieren wir in einem ersten Analyseschritt die EU-Länder nach ihrer Zugehörigkeit zu Parteifamilien. Abbildung 1 zeigt diese Parteienzugehörigkeit nach den vier gängigen, auch vom Manifesto-Projekt verwendeten Zuordnungen. Wir unterscheiden sozialdemokratische, liberale, christdemokratische und konservative/rechte Regierungen (und vernachlässigen Regional- und single-issueParteien). Eine Zuordnung zu einer dieser Parteifamilien erfolgt, wenn eine entsprechende Partei über mehr als die Hälfte der Parlamentssitze aller Regierungsparteien verfügte. Wenn diese Stellung zudem mehr als die Hälfte des jeweiligen Jahres vorherrschte, wurde die Zelle für das betreffende Jahr und Land entsprechend schat- 
tiert. ${ }^{5}$ Wenn es keine dominante Regierungspartei gab, errechneten wir den tagesgewichteten Durchschnitt der parteispezifischen Parlamentssitzanteile. Wenn auch dieses Verfahren keine Zuordnung erlaubte, wurde die Zelle in Abbildung 1 entsprechend markiert.

Aus Abbildung 1 sind mehrere wichtige Trends unmittelbar ersichtlich. Bedeutsam auch für die weitere Analyse ist zunächst die christdemokratische Hegemonie in den 1950er und 1960er Jahren. Als zweite wichtige Erkenntnis zeigt sich, dass jeder Erweiterungsschritt die EU »linker« werden ließ. Schließlich ist aus Abbildung 1 auch die Dominanz konservativer Parteien in den EU-Ländern in den 1980er Jahren ersichtlich, während die 1990er Jahre dann eine deutliche Linksverschiebung mit sich brachten.

In einer nächsten, etwas feinkörnigeren Betrachtung können wir fragen, wie sich Veränderungen in der parteipolitischen Regierungszusammensetzung in Veränderungen im parteipolitischen Gravitationszentrum des EU-Ministerrats übersetzt haben. Für diesen Zweck nehmen wir aus den Daten von Laver/Hunt (1992) die Angaben zur »increase service vs. cut taxes«-Frage, die eine Verortung von Parteien auf der sozioökonomischen Links-rechts-Skala mit Werten zwischen eins und 20 ermöglicht. Die Einschätzungen für einzelne Parteien fließen dann gewichtet nach dem parteispezifischen Sitzanteil an den Sitzen aller Regierungsparteien in einen aggregierten Positionsindex ein. Das europäische parteipolitische Gravitationszentrum ergibt sich dann als Mittel der Ländergravitationszentren. ${ }^{6}$ Wir sind uns bewusst, dass die Verlängerung der in den frühen 1990er erhobenen Daten zurück in die 1950er Jahre nicht ohne Probleme ist. In diesem Zusammenhang möchten wir erneut betonen, wie wichtig eine vorsichtige Interpretation der Ergebnisse ist, wobei insbesondere auf Veränderungen über Zeit abgestellt werden sollte. Zugleich ist aber festzustellen, dass sich Vertrauen in die Aussagekräftigkeit des Gravitationszentrums aus dem Umstand speist, dass zwischen dem in Abbildung 2 ersichtlichen Trend und den in Abbildung 1 berichteten Veränderungen in der Zusammensetzung der EU-Regierungen ein sehr enger Zusammenhang erkennbar ist.

Welches Bild ergibt sich unter Verwendung der Laver/Hunt-Daten? Sie spiegeln recht deutlich die Rechtsverschiebung zum Ende der 1970er und Beginn der 1980er Jahre wider, die sich in einer Reihe von EU-Ländern vollzog. Die 1980er waren eine eher »konservative« Dekade und es dauerte bis zur zweiten Hälfte der 1990er, bevor linke Parteien in einem nennenswerten Umfang zurück zur Macht fanden. Das linke Zwischenspiel zu Mitte der 1970er Jahre war zu einem erheblichen Teil Resultat der EU-Erweiterung um Irland, Dänemark und Großbritannien im Jahre 1973. In Dänemark und Großbritannien waren entweder bereits zum Beitrittszeitpunkt oder wenig später sozialdemokratische Parteien an der Regierung. Zugleich zeigt Abbildung 2 auch, dass Europa, was die parteipolitische Zusammensetzung seiner Mitgliedsregierungen anbetrifft, stetig heterogener wurde - wie an der ansteigenden Standardabweichung ersichtlich.

5 Bei Koalitionsregierungen unter annähernd gleich großen Partnern entschied die Parteizugehörigkeit des Premierministers über die Zuordnung. Dies war jedoch nur in Belgien zwischen 1995 und 2000 und in Österreich ab dem Jahr 2000 der Fall.

6 Wir haben darauf verzichtet, die Länderwerte nach den Stimmengewichten im Ministerrat zu gewichten, weil wir von dem Einstimmigkeitsprinzip als Standardentscheidungsregel ausgegangen sind. 


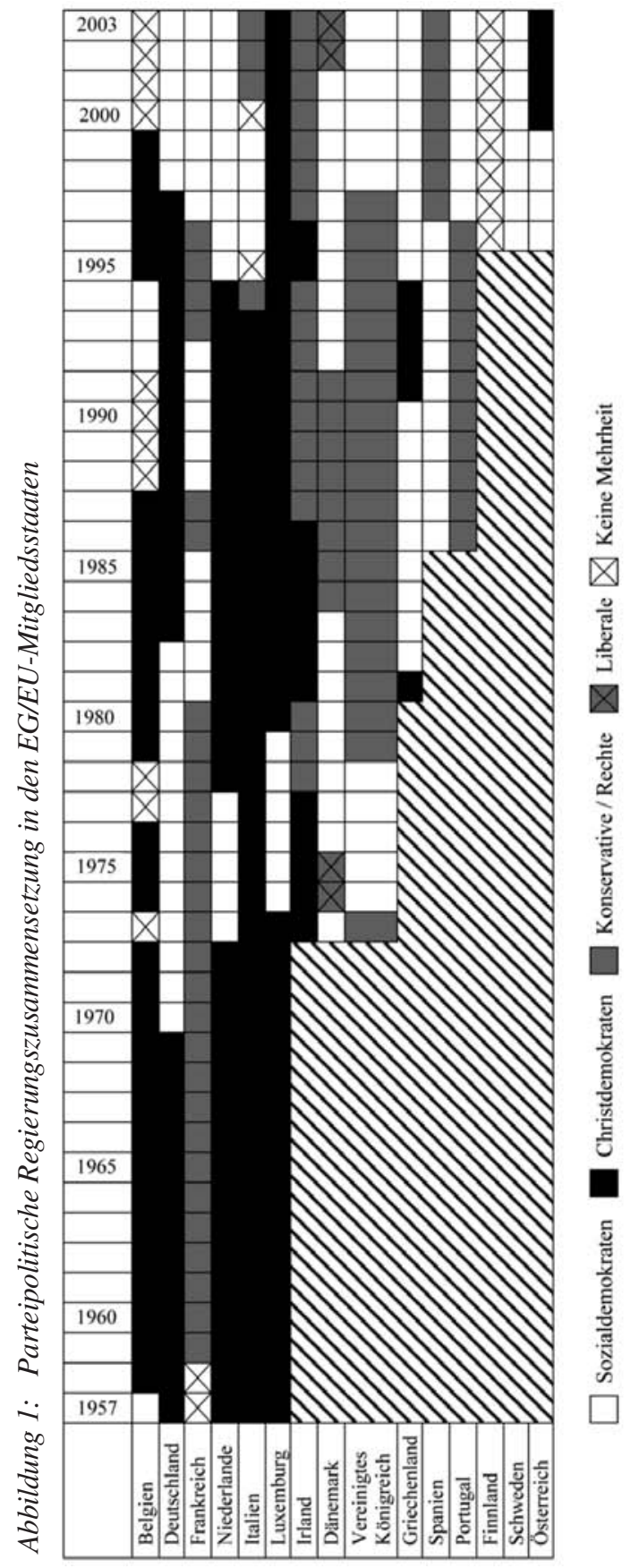


Philip Manow/Armin Schäfer/Hendrik Zorn: Europäische Sozialpolitik in den Jahren 1957-2003

Abbildung 2: Parteipolitisches Gravitationszentrum der Mitgliedsregierungen (Laver/Hunt 1992)

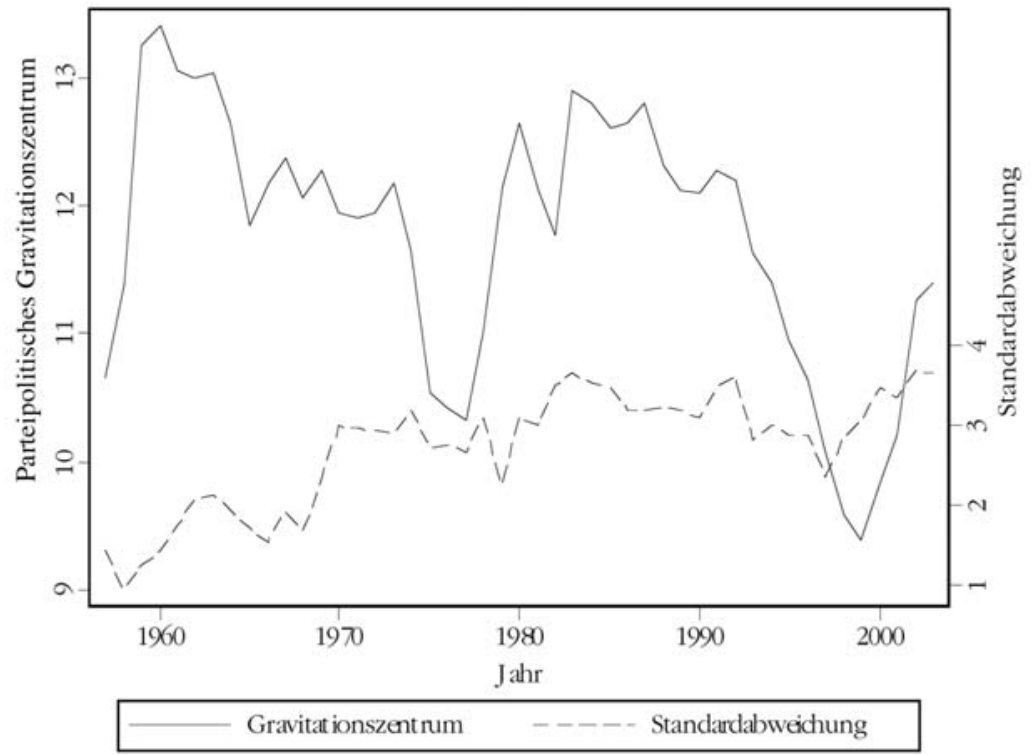

Abbildung 3: Zustimmung der Mitgliedsregierungen zur europäischen Integration (EI) (Ray 1999)

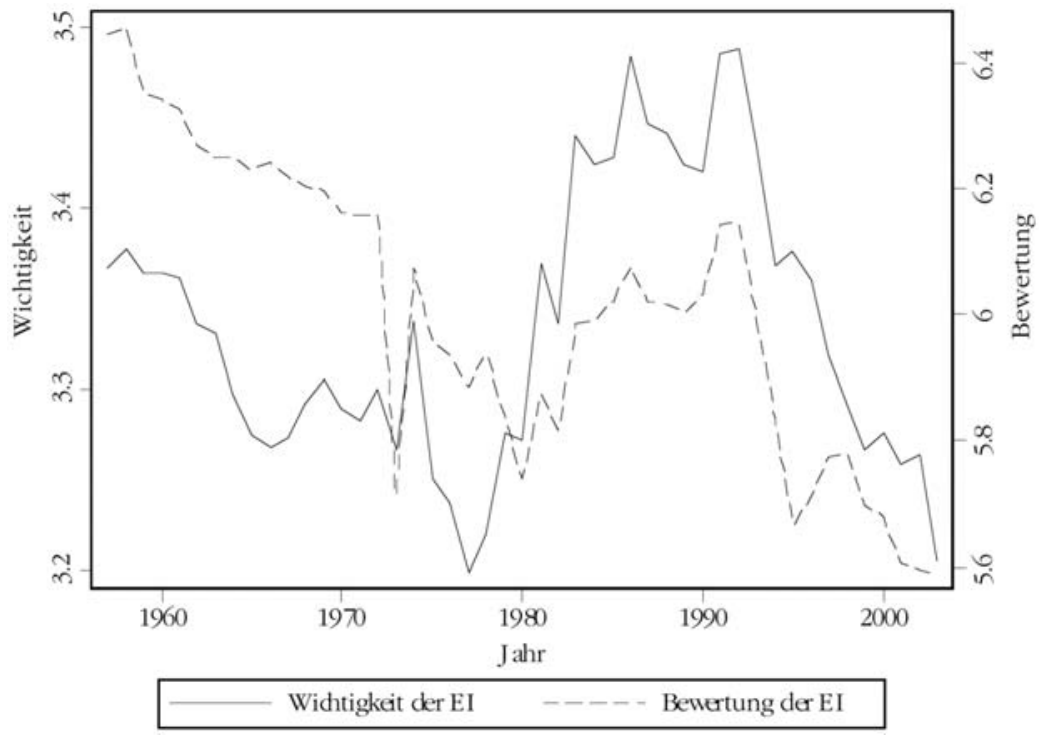


Mit dem gleichen methodischen Vorgehen lässt sich auch die relative Unterstützung des EU-Integrationsprozesses der EU-Mitgliedsregierungen ermitteln. Hier nutzen wir die Daten von Ray (1999), der für die wichtigsten nationalen Parteien ihre Position hinsichtlich der europäischen Integration - ebenfalls per Expertenbefragung - ermittelt hat. Jede Mitgliedslandregierung kann auf einer von eins bis sieben reichenden Integrationsskala verortet werden, je nachdem, ob die Regierungsparteien der Integration der EU »geringe Bedeutung « und »geringe Unterstützung « oder »große Bedeutung « und »große Unterstützung « zumessen. Aus der Verortung der Regierungen in dieser Dimension ergibt sich dann wiederum auf höherer Aggregatstufe die Position der durchschnittlichen Mitgliedslandregierung. Wie Abbildung 3 zeigt, verläuft die Kurve spiegelbildlich zu unserem Gravitationszentrums-Indikator: Je linker die EU-Regierungen waren, umso geringer fällt die Unterstützung für den weiteren Integrationsprozess aus. »Lokale Minima « der Unterstützung finden sich in der zweiten Hälfte der 1970er und der 1990er Jahre. Dass linke Regierungen in der Tendenz integrationsskeptischer sind, zeigt sich auch, wenn wir nach der Unterstützung der EU-Integration unterschiedlicher Parteifamilien fragen (siehe Abb. 4).

Abbildung 4: Zustimmung zur Integration nach Parteifamilien (Ray 1999)

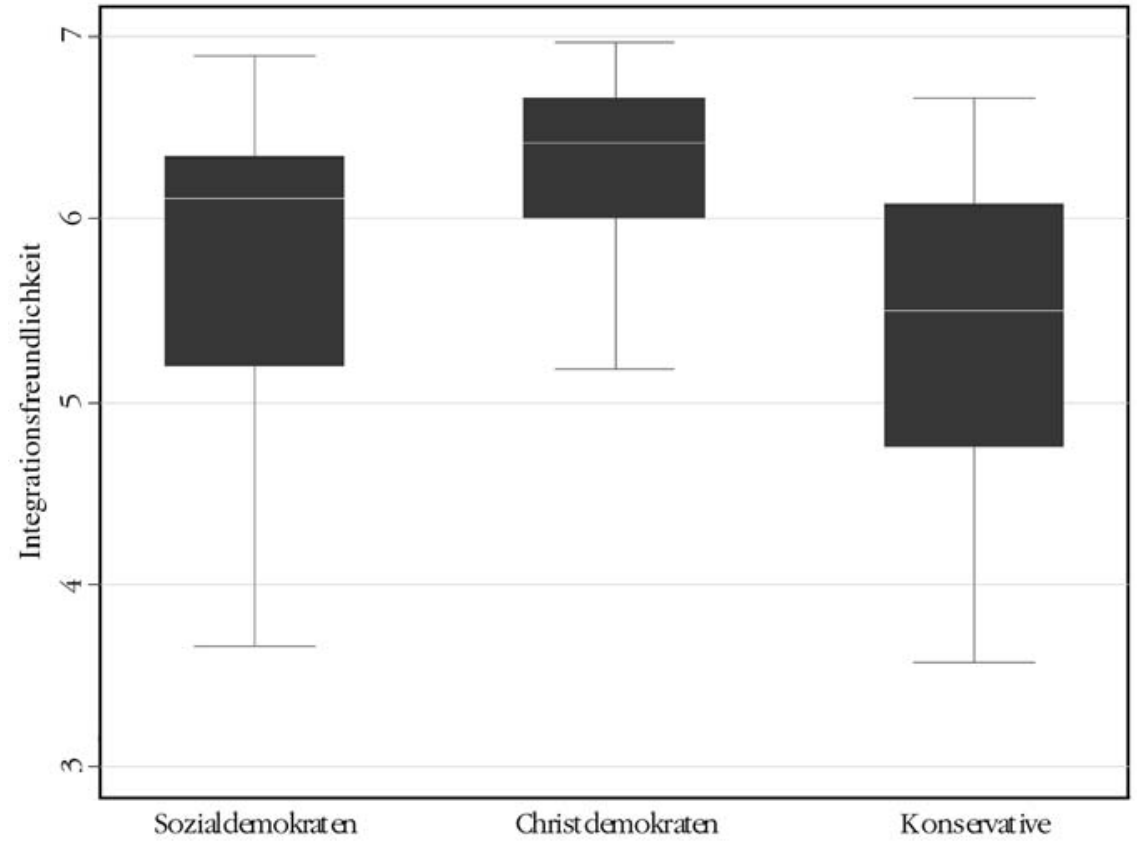

Die box plot-Grafik in Abbildung 4 zeigt die Unterstützung für die Integration der EU, getrennt nach den drei größten Parteifamilien, unter Verwendung der Daten von Ray (1999). Höhere Werte auf der Skala von eins bis sieben stehen für ein stärkeres 
Interesse an der EU und eine größere Unterstützung des Integrationsprozesses. Die box plots zeigen den Median, die gesamte Streuung sowie das erste und vierte Quartil. Wir haben alle Parteien integriert, die sich zumindest einmal seit 1957 in Regierungsverantwortung auf nationaler Ebene befanden und gruppieren diese nach Parteifamilien. Abbildung 4 zeigt, dass zwischen den drei untersuchten großen Parteigruppen erhebliche Unterschiede bestehen. Die christdemokratischen Parteien sind dabei eindeutig die stärksten und geschlossensten Unterstützer der EU. Sozialdemokratische und konservative Parteien sind sich dagegen einig in einer integrationsskeptischeren Haltung und weisen zudem eine höhere interne Heterogenität in dieser Frage auf. Dass dieser »negative Konsens « auch wichtig war für die integrationspolitischen Weichenstellungen, auf die sich die Mitgliedsregierungen in der formativen Phase des europäischen Projekts in den frühen 1950er einigen konnten, zeigen wir im nachfolgenden Abschnitt, in dem es um europäische Sozialpolitik geht. Die Sozialpolitik bietet sich als Gegenstand einer Fallstudie an, weil sozialpolitische Fragen genau an der traditionellen politischen Spannungslinie zwischen »links « und »rechts « liegen (Tsoukalis 1993: 148). In der folgenden Fallstudie betrachten wir die Entwicklung der Sozialpolitik in den Verträgen von Rom bis Amsterdam. Wechselnde ideologische Mehrheiten beeinflussen nicht nur die Wahrscheinlichkeit einer Einigung, sondern schlagen sich auch in den Inhalten der Sozialpolitik nieder. Im Folgenden wird deutlich, wie die Dominanz von Mitte-rechts-Parteien während der entscheidenden Integrationsetappen die EG/EU auf einen ökonomischen Pfad festlegte, auf dem die Sozialpolitik weitgehend untergeordnet blieb.

\section{Parteipolitik und die Europäische Sozialpolitik in den Jahren 1957-2003}

Dieser Abschnitt betrachtet am Beispiel der Sozialpolitik, wie sich Veränderungen der Regierungszusammensetzung auf politische Entscheidungen ausgewirkt haben. Wie aus Abbildung 1 ersichtlich wird, waren die 1960er Jahre von den Christdemokraten dominiert, die 80er und frühen 90er von Mitte-rechts-Koalitionen. Erst in der zweiten Hälfte der 90er Jahre gewinnen die Sozialdemokraten die Oberhand. Alle Vertragsänderungen vor Amsterdam wurden verhandelt, als linke Regierungen in der Minderheit waren. Unser Argument lautet, dass diese Mitte-rechts-Dominanz einer Aufwertung der europäischen Sozialpolitik im Wege stand. Wir folgen einer engen Definition von Sozialpolitik, die nicht jegliche marktkorrigierenden Eingriffe, sondern die »Sicherung gegen die Lebensrisiken einer arbeitsteiligen Gesellschaft « umfasst (Schmidt 2005: 15-16). Die Gemeinsame Agrarpolitik der EU oder die Bildungspolitik bleiben somit außen vor, obwohl sie als präventive Sozialpolitik (im weiten Sinn) verstanden werden können. Im Mittelpunkt stehen die vertraglichen Bestimmungen zur Sozialpolitik im EG-Vertrag. ${ }^{7}$

7 Da die Entwicklung der Sozialpolitik untrennbar mit den großen Konstitutionalisierungsschritten verbunden ist, muss dieser Kontext dargestellt werden. 


\subsection{Die Römischen Verträge}

Als die Europäische Gemeinschaft für Kohle und Stahl (EGKS) 1950/51 gegründet wurde, bestand eine christdemokratische Mehrheit in den sechs Gründungsstaaten. In Belgien, Deutschland, Italien und Luxemburg stellten die Christdemokraten den Regierungschef. In den Niederlanden war die Katholieke Volkspartij stärkste Partei, doch ein Sozialdemokrat (Willem Drees) führte eine große Koalition. Nur in Frankreich war die Situation anders. Die MRP (Mouvement Républicain Populaire) erlangte zu keiner Zeit eine so dominante Stellung wie christdemokratische Parteien in den Nachbarländern (Kalyvas 1996: Kap. 3). In Deutschland, Italien, Luxemburg und den Niederlanden änderte sich bis zum Abschluss der Römischen Verträge nichts an der christdemokratischen Vorherrschaft. Im Gegensatz dazu kam 1954 der Sozialdemokrat Achille von Acker in Belgien an die Macht, wo eine sozialliberale Koalition die Christdemokraten ablöste. In Frankreich regierten von 1956-57 zwei sozialistische Premierminister (Edgar Faure und Guy Molley), die der europäischen Integration weniger skeptisch als ihr Vorgänger Pierre Mendès-France von der Parti Radical gegenüberstanden. Für die Anfangsjahre der europäischen Integration lässt sich dennoch festhalten, dass sie durch christdemokratische Parteien geprägt wurden. Die ideologische Homogenität der beteiligten Regierungen und ihre positive Bewertung des Europagedankens erleichterten den Abschluss der Römischen Verträge.

Auch wenn dies nicht die ursprüngliche Absicht aller Akteure war, glich die Europäische Wirtschaftsgemeinschaft (EWG) im Ergebnis dem (ordo-)liberalen Ideal, dass die Gemeinschaftsinstitutionen zwar das Funktionieren des Markts gewährleisteten, aber keine darüber hinaus gehenden Eingriffsrechte besaßen (von der Groeben 1987: 189; Joerges 2003: 191-192). Sieht man von der Agrarpolitik ab, stützten sich die Römischen Verträge auf die marktwirtschaftlichen Prinzipien eines unverzerrten Wettbewerbs, des Diskriminierungsverbots und der Nicht-Intervention (Ophüls 1961/62: 148-154; Streit/Mussler 1995: 14-15). Sie legten den Grundstein für die Marktintegration, enthielten jedoch kaum Kompetenzen zur nachträglichen Korrektur von Marktergebnissen (Scharpf 1999: 49). Mit der EWG wurde die Wirtschaftsordnung geschaffen, für die sich der ordoliberale Flügel der CDU in Deutschland eingesetzt hatte.

Vor allem Wirtschaftsminister Ludwig Erhard kämpfte innenpolitisch gegen den aus seiner Sicht übermäßigen Ausbau des Sozialstaats, der seinen Höhepunkt in der Rentenreform 1957 fand (Abelshauser 1996: 384-390). Im selben Jahr mussten Erhard und seine ordoliberalen Mitstreiter eine stark abgeschwächte Version der Kartellgesetzgebung hinnehmen. Auch aufgrund dieser innenpolitischen Niederlagen stritt der Wirtschaftsminister für eine liberale internationale Wirtschaftsordnung. Dies brachte ihn dazu, den britischen Vorschlag einer OEEC-Freihandelszone statt der Europäischen Zollunion der EGKS-Staaten zu unterstützen. Eine »kleineuropäische« Lösung barg aus seiner Sicht das Risiko der gemeinsamen Abschottung nach außen. Erhard konnte sich mit seinem Ansinnen nicht gegen Kanzler Konrad Adenauer durchsetzen, der dem politischen Ziel der engen Zusammenarbeit 
mit Frankreich Vorrang vor einer primär ökonomischen Logik einräumte (Lee 1995: 43-44). Nach dieser Vorentscheidung für das Europa der Sechs verschob sich das Ziel für die Ordoliberalen dahingehend, in den Verhandlungen über den Gemeinsamen Markt interventionistische Versuchungen abzuwehren. Die deutsche Verhandlungsposition im Vorfeld der Römischen Verträge lässt sich als CDU-interner Kompromiss zwischen den protestantischen, marktliberalen »Transatlantikern « und den katholischen, sozialstaatlichen »Frankophilen« deuten.

Dieser Kompromiss führte dazu, dass eine Reihe liberaler deutscher Juristen und Ökonomen trotz Erhards ursprünglicher Bedenken entscheidend auf die Wirtschaftsordnung der EWG Einfluss nahm (Gerber 1994: 71-72). Hans von der Groeben, Mitglied des Spaak-Komitees, sicherte die Unterstützung einer Mehrheit der Mitglieder für eine marktwirtschaftliche Ordnung des Gemeinsamen Markts gegen stärker dirigistische Vorstellungen italienischer und französischer Delegierter (Küsters 1989: 86). Schon zu Beginn der Verhandlungen in diesem Ausschuss legte Erhards Staatssekretär Alfred Müller-Armack einen einflussreichen Textentwurf vor, der in der Folge die Diskussion anleitete. Mehrere Mitarbeiter des Wirtschaftsministeriums, die ihr Engagement für Europa mit liberalen Überzeugungen verbanden, sahen die EWG als Möglichkeit, die innenpolitisch verwässerten Wettbewerbsregeln europäisch durchzusetzen (Hentschel 1998: 380). Natürlich waren sie nicht auf ganzer Linie erfolgreich, doch gelang ihnen die Beschränkung der Handlungsbefugnisse für marktverzerrende Eingriffe (Küsters 1982: 266, 305). Zwar erhielten die Wirtschaftsordnung und die vier Freiheiten des EWG-Vertrags erst durch die Rechtsprechung des Europäischen Gerichtshofs (EuGH) ihre Durchschlagskraft, dennoch konnte der EuGH seine Interpretation der Verträge auf die darin enthaltene Ordnungspolitik ordoliberaler Provenienz stützen. ${ }^{8}$ Auch wenn diese Entwicklung 1957 nicht im Einzelnen vorhergesehen worden war (Ehlermann 1995: 84-85), begrüßte sie Müller-Armack (1966: 405) rückblickend als »List der Idee«.

Der Schwerpunkt vor Abschluss der Römischen Verträge lag auf wirtschaftlichen Themen. Dennoch gab es um die Sozialpolitik eine Auseinandersetzung, die vor allem zwischen der französischen und der deutschen Regierung ausgetragen wurde. Dies überrascht nicht, denn die Parteien mit der größten Distanz zum errechneten Gravitationszentrum sind die pivotalen Akteure in Verhandlungen. Ihre Zustimmung muss gewonnen werden. Die französischen Sozialisten und der ordoliberale CDUFlügel stellten in den Verhandlungen die ideologischen Gegenpole zueinander dar. Um dem Entwurf zustimmen zu können, verlangte die französische Regierung, dass die Verträge ein Mandat zur sozialpolitischen Harmonisierung enthielten. Der französische Premierminister Guy Mollet befürchtete Wettbewerbsnachteile durch die teilweise noch auf Leon Blums Front Populaire-Regierung zurückgehende Sozialgesetzgebung. In Frankreich existierten eine gesetzlich geregelte Vierzig-StundenWoche, bezahlter Urlaub, Überstundenzuschläge sowie gleiche Bezahlung für Män-

8 »Der wichtigste deutsche Beitrag zur Ausgestaltung der EWG bestand in der ordnungstheoretischen Grundlegung und wirtschaftsverfassungsrechtlichen Konkretisierung der für die Wirtschaftsgemeinschaft maßgeblichen Grundsätze« (Mestmäcker 2003: 290). 
ner und Frauen. Deshalb forderte Mollet in diesen Bereichen die Harmonisierung der Vorschriften, um einen fairen Wettbewerb in der Zollunion sicherzustellen. Das Motiv hierbei war jedoch weniger das Streben nach einem europäischen Sozialstaat als das Angleichen der Kosten für Produzenten verschiedener Länder.

Doch selbst eine begrenzte Vereinheitlichung der Sozialpolitik stieß auf den Widerstand der deutschen Verhandlungsdelegation und des Wirtschaftsministers (Milward 2000: 213-214). Die Entscheidung in dieser Frage lieferte Adenauers Frankreichbesuch auf dem Höhepunkt der Suezkrise am 6. November 1956: Während der Vertrag einige Klauseln zur Sozialpolitik enthalten würde, beschränkten sich diese überwiegend auf rechtlich nicht bindende Absichtserklärungen. ${ }^{9}$ Dieser Kompromiss war ein im Wesentlichen symbolischer Sieg Mollets, der es ihm erleichtern sollte, die benötigte Mehrheit zur Ratifizierung der Römischen Verträge zu gewinnen (Lynch 1994: 84; 1997: 181).

Schließlich waren zwölf von 248 Artikeln des EWG-Vertrags der Sozialpolitik gewidmet. Artikel 118 trug der Europäischen Kommission auf, eine »enge Zusammenarbeit « in der Sozialpolitik zu fördern, vor allem in den Bereichen Beschäftigung, Arbeitsrecht und Arbeitsbedingungen, berufliche Aus- und Fortbildung, soziale Sicherheit, Verhütung von Berufsunfällen und -krankheiten, Gesundheitsschutz bei der Arbeit sowie Koalitionsrecht und Koalitionsverhandlungen zwischen den Sozialpartnern. Diese Ziele sollten allerdings nicht durch Kompetenztransfers auf die europäische Ebene oder die Schaffung sozialer Rechte erzielt werden. Stattdessen war die Kommission aufgefordert, sie durch »Untersuchungen, Stellungnahmen und die Vorbereitung von Beratungen « zu fördern. Mehr Biss hatte Artikel 119, der den Grundsatz des gleichen Entgelts für Männer und Frauen festschrieb. In den siebziger Jahren führte diese Bestimmung $\mathrm{zu}$ einer Reihe wichtiger Urteile des EuGH, die einige Mitgliedsstaaten zu erheblichen Anpassungen zwang (am Beispiel Großbritanniens Alter/Vargas 2000: 457-468).

Das Verständnis von Sozialpolitik in den Römischen Verträgen hing eng mit der Schaffung eines grenzüberschreitenden Arbeitsmarkts zusammen. Der Europäische Sozialfonds (Art. 123-128) zielte deshalb neben der Erleichterung des Strukturwandels auf die Verbesserung von Beschäftigungsfähigkeit und Mobilität der Arbeitskräfte durch Umschulungen und Umsiedlungsbeihilfen (Art. 125a). Neben diesen an den Gemeinsamen Markt gekoppelten Vorschriften gab es keine eigenständige Sozialpolitik. Im Ergebnis erhielt die EWG Kompetenzen, Märkte zu schaffen, nicht aber Marktergebnisse zu korrigieren. Die Verantwortung dafür verblieb bei den Mitgliedsstaaten. Der nicht beschrittene sozialpolitische Harmonisierungspfad (»the road not taken«) ermöglichte erst, wie Fritz Scharpf (2002: 646) hervorhebt, die anschließende Entkoppelung wirtschaftlicher Integration von Fragen des sozialen Schutzes.

Zusammenfassend lässt sich für diese frühe Phase festhalten, dass ein parteipolitischer Ansatz zwar nicht die Motivation für die Integration vollständig erklären

9 Aufschlussreich hierzu Carstens (1976: 599), der Adenauer nach Frankreich begleitete und zusammen mit Robert Marjolin die Kompromissformel für die umstrittenen Passagen formulierte. 
kann, aber dennoch wichtige Hinweise dafür liefert, weshalb der Weg sozialpolitischer Harmonisierung nicht bestritten wurde. Der Blick auf das parteipolitische Gravitationszentrum verdeutlicht, weshalb stattdessen der Pfad wirtschaftlicher Integration eingeschlagen wurde: Christdemokratische Regierungsparteien begrüßten die wirtschaftliche Integration, sahen aber keine Notwendigkeit für eine begleitende Harmonisierung der Sozialpolitik.

\subsection{Relance européenne: Die Einheitliche Europäische Akte}

Mitte der achtziger Jahre fand mit der Einheitlichen Europäischen Akte (EEA) die erste Reform der Gründungsverträge statt. Nach einem »Linksschwenk « in den siebziger Jahren, führten Regierungswechsel Anfang der Achtziger zu einer deutlichen Rechtsverschiebung des Gravitationszentrums. Zudem war die EG durch neue Mitglieder politisch und sozioökonomisch heterogener geworden (Abb. 1 und 2). Aus parteipolitischer Perspektive erschien es deshalb unwahrscheinlich, dass die Mitgliedsstaaten sich auf eine Neubelebung der Integration einigen würden. Noch unwahrscheinlicher waren sozialpolitische Initiativen. Die britische Regierung verfolgte innenpolitisch ein Programm der Liberalisierung, Privatisierung und Deregulierung. Ihren Gegenpol fand Margaret Thatcher im französischen Präsidenten François Mitterrand und in der an John Maynard Keynes orientierten interventionistischen Politik der französischen Regierung. Bis 1983 war eine Einigung auf ein gemeinsames europäisches Projekt dieser beiden Regierungen ausgeschlossen $-\mathrm{zu}$ groß war ihre ideologische Distanz. Erst nachdem das »französische Experiment« gescheitert war, eröffnete sich die Möglichkeit eines Neuanfangs. Mitterrand versuchte nun, die neue französische Wirtschaftspolitik europäisch abzusichern, indem er selbst zum Verfechter von Marktintegration und Liberalisierung in der EG wurde (Keohane/Hoffmann 1990: 287-288; Ross 1995: 258, Fn. 52). Damit traf er den Nerv der Mitte-rechts-Koalitionen anderer Mitgliedsstaaten. Die Entscheidung für die Vollendung des Binnenmarkts mit der EEA reflektierte die dort innenpolitisch vielfach schon vollzogene Hinwendung zum Markt (Hall 1999: 154).

Die Einheitliche Europäische Akte wurde 1986 von zwölf Staaten unterschrieben. Zwischen 1983-1987 bestand eine klare Mehrheit von Mitte-rechts-Regierungen in Europa. 1985 waren sieben von damals noch zehn Premierministern Christdemokraten oder Konservative (in Belgien, Dänemark, Deutschland, Irland, Luxemburg, den Niederlanden und Großbritannien). In Italien war die Democrazia Cristiana noch immer die stärkste Partei, aber zum ersten Mal seit dem Zweiten Weltkrieg konnten die Sozialisten (Partito Socialista Italiano) mit Bettino Craxi den Regierungschef stellen. In Frankreich und Griechenland gab es linke Mehrheiten. Ein Jahr später hatte sich nur in Frankreich die Situation verändert, wo die erste Phase der Kohabitation stattfand. In den neuen südeuropäischen Mitgliedsländern regierten die Sozialisten (Partido Socialista Obrero Español) in Spanien, die Konservativen (Partido Social Democrata) in Portugal. 
In der Literatur bestehen verschiedene Erklärungen für die Wiederbelebung der Integration in den achtziger Jahren. Andrew Moravcsik (1998: 317, 332-335) verweist auf die Konvergenz wirtschaftspolitischer Interessen in Deutschland, Frankreich und Großbritannien, die nach 1983 möglich wurde. Im Gegensatz dazu hebt Green Cowles (1995) vor allem den Einfluss von Großunternehmen durch den European Round Table of Industrialists hervor. Schließlich begreifen Wayne Sandholtz und John Zysman (1989) die Kommission als politischen Unternehmer (policy entrepreneur), der durch sein Verhandlungsgeschick einen Erfolg erst ermöglichte. Wir bestreiten nicht, dass diese Faktoren das Ergebnis beeinflusst haben. Allerdings beachten sie aus unserer Sicht wichtige politische Variablen - nämlich veränderte Mehrheiten und ideologische Konvergenz - zu wenig. Die Dominanz von Mitterechts-Regierungen, die größere Bedeutung, die diese Parteien der Integration zumaßen (Abb. 3), sowie ihre wachsende Marktorientierung (Abb. 5) erleichterten die Entscheidung, den Binnenmarkt zum Leitstern vertiefter Integration zu machen. Eine politisch heterogenere Gruppe der Staats- und Regierungschefs hätte eine Einigung dagegen erschwert. Das bedeutet freilich nicht, dass in den Verhandlungen nationale Interessen keine Rolle spielten, aber sie sind nicht einfach der Spiegel nationaler Wirtschaftsinteressen, sondern parteipolitisch »eingefärbt«.

\section{Abbildung 5: Befürwortung marktliberaler Positionen}

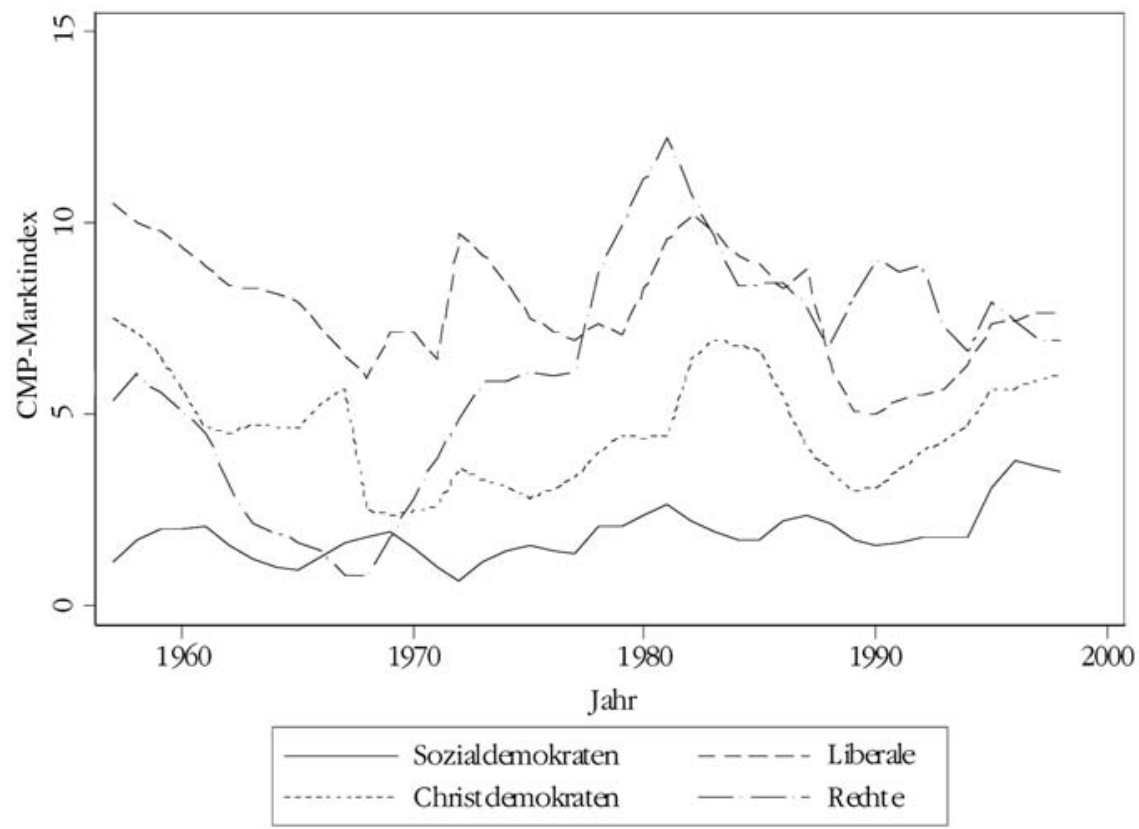


Möchte man nun den sozialpolitischen Gehalt der EEA verstehen, ist zunächst zu beachten, dass die Vertragsänderungen die Herstellung des Binnenmarkts zum Kern hatten. Bis 1992 sollten die vier Freiheiten (Freizügigkeit für Arbeit, Dienstleistungen, Güter und Kapital) verwirklicht werden. Aus vier Gründen war diese Entscheidung bemerkenswert. Erstens, indem ein Datum - »1992« - festgelegt wurde, gab es eine klare Zielmarke, die es in relativ kurzer Zeit zu erreichen galt. Zweitens wurde die politische Entscheidung für Liberalisierung und den Abbau von verbleibenden Handelsschranken als vermeintlich technische Angelegenheit präsentiert. Die Europäische Kommission legte einen Katalog von über 300 Einzelmaßnahmen zur Verwirklichung des Binnenmarkts vor. Da die Bedeutung einzelner Maßnahmen nur für Experten kenntlich war, ließ sich kaum eine öffentliche Debatte darüber führen. Drittens einigten sich die Staats- und Regierungschefs darauf, zukünftig in den Binnenmarkt betreffenden Fragen Mehrheitsabstimmungen zuzulassen. Sie akzeptierten den Verlust von Souveränität, um die Märkte von ihren Fesseln zu befreien. Schließlich stand, viertens, mit der »gegenseitigen Anerkennung «, die 1979 im berühmten Cassis de Dijon-Urteil vom EuGH durchgesetzt worden war (siehe Alter/MeunierAitsahalia 1994: 539-540), ein Instrument zur Verfügung, das Marktbarrieren ohne weitere politische Entscheidungen überwinden half (Streeck 1998: 374-375).

In der Sozialpolitik enthielt die Einheitliche Europäische Akte zwei Änderungen: Die Artikel 118a und 118b wurden eingeführt. Der erste Artikel führte qualifizierte Mehrheitsentscheidungen in die europäische Sozialpolitik ein. Die Mitgliedsstaaten wurden aufgefordert, Richtlinien zur Verbesserung der Arbeitsumwelt zu verabschieden, um die Sicherheit und Gesundheit der Arbeitnehmer zu schützen. Da die Formulierung vage blieb, ermöglichten diese Artikel der Kommission das »treatybase game «, mit dem sie die Anwendung der Mehrheitsregel ausweiten wollte (Rhodes 1998: 122-128). Artikel 118b forderte rechtlich unverbindlich die Entwicklung des Sozialen Dialogs auf europäischer Ebene. Er führte die ergebnislosen Val Duchesse-Gespräche fort, die Mitte der 1980er Jahre initiiert worden waren (Ross 1995: 38). Trotz Delors' Werben für einen »Europäischen Sozialraum« blieb der sozialpolitische Inhalt der EEA marginal.

Die achtziger Jahre endeten mit einer feierlichen Erklärung des Europäischen Rats zur Charta der Grundrechte der Arbeitnehmer (Sozialcharta). Dieser Versuch, soziale Rechte zu institutionalisieren, blieb - vor allem aufgrund britischen Widerstands - nicht nur unverbindlich, sondern wurde zudem auf die Arbeitnehmer begrenzt. Während der Wechsel in der französischen Wirtschaftspolitik einen pivotalen Akteur an die Mehrheitsposition heranführte, blieb eine solche Wende in der Sozialpolitik aus. Die britischen Konservativen wehrten selbst zaghafte Versuche einer europäischen Sozialpolitik ab.

\subsection{Maastricht: Währungsunion und Sozialprotokoll}

Mit der Währungsunion entschieden sich die EG-Staaten für eine beispiellose Abgabe von Souveränität. Sie gaben nicht nur die Geld- und Wechselkurspolitik als 
Instrumente nationaler Wirtschaftspolitik auf, sondern schufen darüber hinaus mit der Europäischen Zentralbank einen Akteur, dessen Unabhängigkeit von politischen Entscheidungen ausgeprägter als die der Deutschen Bundesbank war (Scharpf 1999: 29-30). Die Stabilität der gemeinsamen Währung sollte zusätzlich durch sanktionierbare Regeln für die Haushaltspolitik abgesichert werden. Neben diesem weitgehenden Transfer von Entscheidungsbefugnissen nahm sich die Entwicklung der Sozialpolitik bescheiden aus. Aufgrund erneuten britischen Widerstands konnten die vorgesehenen Änderungen nicht Teil des Vertrags werden, sondern mussten in einem Zusatzprotokoll festgehalten werden, das es den elf anderen Unterzeichnerstaaten des »Abkommens über die Sozialpolitik« erlaubte, die Mehrheitsregel auf weitere Bereiche der Sozialpolitik anzuwenden. Das britische »Opt-out « konnte erst sechs Jahre später mit dem Amsterdamer Vertrag aufgehoben werden - nachdem sich die politischen Mehrheitsverhältnisse in Europa und in Großbritannien gedreht hatten.

Dem Anschein nach lässt sich der Maastrichter Vertrag gut aus parteipolitischer Perspektive erklären. 1991 hatten zehn von zwölf Mitgliedsstaaten konservative oder christdemokratische Regierungschefs (Belgien, Dänemark, Deutschland, Griechenland, Irland, Italien, Luxemburg, die Niederlande, Portugal und Großbritannien). ${ }^{10}$ Nur Frankreich und Spanien wurden von Sozialisten/Sozialdemokraten regiert (Abb. 1). Von den beiden Letztgenannten befürwortet Frankreich die Währungsunion, um einerseits das wiedervereinigte Deutschland einzubinden und andererseits Einfluss auf die Geldpolitik zurück zu gewinnen (Dyson/Featherstone 1999: 83-85, 757). Nimmt man also die Lage des politischen Gravitationszentrums, die Prominenz integrationsfreundlicher christdemokratischer und marktorientierter konservativliberaler Regierungen zusammen, wird sowohl plausibel, weshalb die Wahrscheinlichkeit einer politischen Einigung Anfang der neunziger Jahre besonders hoch war, als auch, weshalb der Schwerpunkt erneut auf wirtschaftlicher Integration lag. In Antizipation späterer Regierungswechsel einigten sich die Staats- und Regierungschefs mit der Währungsunion auf rechtlich bindende Verfahren und autonome Institutionen, die weniger disziplinierten Staaten (oder Regierungsparteien) die Hände binden sollten. Sie nutzten damit die parteipolitisch »günstige Situation« zur unumkehrbaren Verankerung ihrer wirtschaftspolitischen Präferenzen. Allerdings unterzeichneten in Maastricht elf der zwölf Mitgliedsstaaten ebenfalls das Abkommen über die Sozialpolitik. Angesichts der Dominanz von Mitte-rechts-Regierungen muss die Frage beantwortet werden, weshalb sie dies taten. Zunächst sollen die Inhalte des Abkommen dargestellt werden, bevor wir zu dieser Frage zurückkehren.

Das Abkommen über die Sozialpolitik enthielt drei bemerkenswerte Veränderungen (hierzu Kowalsky 1999: 153-154). Erstens weitete es die sozialpolitische Zuständigkeit der Gemeinschaft auf folgende Bereiche aus:

10 Karl Magnus Johansson hebt hervor, dass vor allem die Christdemokraten den Weg nach Maastricht bereiteten: »Christian Democrats dominated the three presidencies charged with the task of initiating and completing the two IGCs. During the second half of 1990 Italy held the Presidency, followed by Luxembourg and the Netherlands « (Johansson 2002: 876). 
- Verbesserung der Arbeitsumwelt zum Schutz von Sicherheit und Gesundheit der Arbeitnehmer;

- Arbeitsbedingungen;

- Unterrichtung und Anhörung der Arbeitnehmer;

- berufliche Eingliederung der aus dem Arbeitsmarkt ausgegrenzten Personen;

- Chancengleichheit von Männern und Frauen auf dem Arbeitsmarkt und Gleichbehandlung am Arbeitsplatz;

- soziale Sicherheit und sozialer Schutz der Arbeitnehmer;

- Schutz der Arbeitnehmer bei Beendigung des Arbeitsvertrags;

- Vertretung und kollektive Wahrnehmung der Arbeitnehmer- und Arbeitgeberinteressen, einschließlich der Mitbestimmung;

- Beschäftigungsbedingungen der Staatsangehörigen dritter Länder, die sich rechtmäßig im Gebiet der Gemeinschaft aufhalten;

- finanzielle Beiträge zur Förderung der Beschäftigung und zur Schaffung von Arbeitsplätzen, und zwar unbeschadet der Bestimmungen über den Sozialfonds.

Ausdrücklich ausgeschlossen wurden jedoch das Arbeitsentgelt, das Koalitions-, Streik- und Aussperrungsrecht. Durch das Sozialabkommen konnten - zweitens - in den ersten fünf Bereichen Richtlinien mit qualifizierter Mehrheit verabschiedet werden. Das Einstimmigkeitsprinzip galt jedoch weiter für die Bereiche sechs bis zehn. Schließlich sah Artikel vier des Abkommens vor, dass der Soziale Dialog auf Gemeinschaftsebene »zur Herstellung vertraglicher Beziehungen, einschließlich des Abschlusses von Vereinbarungen, führen [kann] «. Das heißt, neben den legislativen Pfad zu sozialpolitischen Richtlinien trat ein korporatistisches Verfahren, das den Sozialpartnern Gesetzgebungskompetenz zusprach (Falkner 1998: 82-84). Das Abkommen über die Sozialpolitik enthielt also eine moderate Ausweitung der Sozialpolitik, doch die Einführung von Sozialpartnerabkommen war eine beachtenswerte Neuerung. Sein größter Schwachpunkt war, dass Großbritannien von allen verabschiedeten Richtlinien ausgenommen sein würde und somit die Reichweite europäischer Sozialpolitik begrenzt blieb. Doch unabhängig von dieser Bewertung müssen wir fragen, weshalb das Sozialabkommen überhaupt verabschiedet wurde. Drei Punkte sind hervorzuheben:

Erstens weist Abbildung 3 darauf hin, dass die hohe Zustimmung zur Integration Anfang der neunziger Jahre die Verhandlungen in Maastricht erleichterte - zumindest solange die Briten von einer Totalblockade abgehalten werden konnten. Getragen wurden die hohen Zustimmungswerte von den stark vertretenen, integrationsfreundlichen christdemokratischen Parteien. Obwohl die Christdemokraten die Währungsunion als Kernprojekt weiterer Integration ansahen, unterstützten sie ebenfalls die behutsame Ausweitung einer arbeitsmarktnahen Sozialpolitik (Johansson 2002: 886). Die Trennlinie verlief in dieser Frage zwischen den Christdemokraten auf der einen und den britischen Konservativen auf der anderen Seite, da Premierminister John Major einen symbolischen Erfolg benötigte, um seine Position innerhalb der eigenen Partei zu stärken (Lange 1993: 25-27; Pierson 1996: 154155). Major war zur Verhinderung jeglicher sozialpolitischer Initiativen entschlossen - und dies, obwohl selbst die britische Industrie die verbliebenen sozialpoliti- 
schen Vorschläge als eine »relatively minor affair« ansah (Moravcsik 1998: 422). ${ }^{11}$ Zweitens, den Sozialpartnern das Recht zuzugestehen, autonom über SozialpolitikRichtlinien zu verhandeln, war gefahrlos, weil die Arbeitgeber kein Interesse an solchen Verhandlungen hatten (Streeck 1998: 388). Drittens, auch mit dem Abkommen über die Sozialpolitik blieb die europäische Sozialpolitik in ihrer Reichweite begrenzt und eng an die Wirtschaftsintegration gekoppelt. Selbst mit Maastricht blieben die EU-Befugnisse in der Sozialpolitik auf Teile des Arbeitsrechts konzentriert. Die neuen Kompetenzen glichen darüber hinaus nicht den Verlust nationaler Autonomie aus, der mit negativer Integration und erhöhtem Wettbewerb einherging. In der Summe verringerte sich die sozialpolitische Gestaltungsfähigkeit der Mitgliedsstaaten (Leibfried/Pierson 2000: 287-288).

Das parteipolitische Gravitationszentrum kann zwar die Verhandlungsergebnisse nicht exakt vorhersagen, aber es umreißt den Raum möglicher Kompromisse und verdeutlicht, welche Akteure auf Ausgleichszahlungen dringen können. ${ }^{12}$ Die Christdemokraten waren in Maastricht in einer starken Verhandlungsposition, und das Ergebnis - die Währungsunion sowie eine moderate Ausweitung der Sozialpolitik - entsprach weitgehend ihren Zielen (Hix/Lord 1997: 189). Im Umkehrschluss bedeutet dies, dass eine andere politische Mehrheit entweder zum Scheitern der Verhandlungen oder zu einem anderen Ergebnis geführt hätte. Aber erst nach Maastricht sollte sich das Gravitationszentrum nach links verschieben. In Amsterdam gab es zum ersten Mal in der Geschichte der europäischen Integration eine Mehrheit links von der Mitte (d. h. einen Wert unter zehn für das Gravitationszentrum in Abb. 2) doch inzwischen hatte sich die Sozialdemokratie selbst nach rechts bewegt.

\subsection{Das sozialdemokratische Zwischenspiel: Amsterdam und Lissabon}

Zwischen 1997 und 2002 regierten mehr sozialdemokratische Parteien in der EU als jemals zuvor. Für eine kurze Zeitspanne beherrschten sie die europäische Agenda. Die Wahlerfolge der Sozialdemokratie führten in Amsterdam (1997) einerseits zur Integration des Abkommens über die Sozialpolitik und andererseits zur Aufnahme eines neuen Titels »Beschäftigung « in die Verträge. Drei Jahre später beschlossen die Staats- und Regierungschef die so genannte Lissabon-Strategie und führten die »Offene Methode der Koordinierung « ein. ${ }^{13}$ Während in dieser Zeit ehrgeizige Ziele

11 Offensichtlich bestimmte die Politik hierbei ganz wesentlich das »nationale Interesse «. Eher unwahrscheinlich erscheint, dass eine Labour-Regierung den Zwang verspürt hätte, vermeintliche Wirtschaftsinteressen energischer zu vertreten als der Unternehmensverband CBI (Confederation of British Industry). Es ist daher recht einfach, das »counterfactual« zu formulieren: Hätte Labour regiert, wäre die Sozialpolitik offizieller Bestandteil der Verträge geworden.

12 Moravcsik (1998: 453) argumentiert, dass die ärmeren Mitgliedsstaaten das Sozialprotokoll aufgrund von Ausgleichszahlungen akzeptierten. Im Fall von Spanien konnte Delors zudem an die Solidarität einer sozialdemokratischen Regierung appellieren.

13 Im Folgenden verwenden wir die gebräuchlichere englische Abkürzung »OMC« für Open Method of Coordination. 
vereinbart wurden, blieben sie jedoch rechtlich unverbindlich. Stattdessen wurde mit der Europäischen Beschäftigungsstrategie - einem Vorläufer der OMC - ein weiches Koordinierungsverfahren eingeführt, das gemeinsame Zielvereinbarungen und wechselseitiges Lernen an die Stelle sanktionierbarer Regeln setzte. Zwei Faktoren erklären dieses Ergebnis: Zum einen waren die nun regierenden Sozialdemokraten viel weniger interventionistisch als dies noch in den siebziger oder achtziger Jahren der Fall gewesen war. Zum anderen implodierten die Zustimmungswerte zur Integration nach Maastricht. Beide Faktoren führten dazu, dass die sozialdemokratischen Regierungen in Amsterdam von einer Ausweitung sozialpolitischer Befugnisse der EU absahen. Damit gelang es ihnen nicht, Institutionen zu schaffen, die ihre Nachfolger in ähnlicher Weise wie die Währungsunion binden würden.

Wie wir im vorangegangenen Abschnitt gesehen haben, dominierten Mitte-rechtsRegierungen die Maastrichter Verhandlungen. Mitte der neunziger Jahre begannen sich die Mehrheitsverhältnisse zu ändern und das parteipolitische Gravitationszentrum verschob sich nach links. Mit der Norderweiterungsrunde (Finnland, Schweden, Österreich) traten zudem drei Länder mit starken sozialdemokratischen Parteien der EU bei. Während des Amsterdamer Gipfels waren schließlich zehn von 15 Premierministern Sozialisten/Sozialdemokraten (Dänemark, Finnland, Frankreich, Griechenland, Großbritannien, Italien, die Niederlande, Österreich, Portugal und Schweden). ${ }^{14}$ In Belgien und Luxemburg führten Christdemokraten große Koalitionen. Nur Deutschland, Irland und Spanien hatten Mitte-rechts-Regierungen. Vielleicht noch wichtiger als die bloße numerische Überlegenheit war der Zeitpunkt der Wahlen in Frankreich und Großbritannien. Tony Blair und Lionel Jospin wurden wenige Wochen vor dem Gipfeltreffen ins Amt gewählt. Während ihre Vorgänger eine Ausweitung sozialpolitischer Kompetenzen strikt abgelehnt hatten, unterstützten die neu gewählten Regierungen dies. So hatte Blair schon vor der Wahl angekündigt, dass Großbritannien das Abkommen über die Sozialpolitik unterzeichnen würde. ${ }^{15}$

Das wichtigste Ergebnis der parteipolitischen Linksverschiebung war die Aufnahme des Sozialabkommens sowie des neuen Titels zur Beschäftigung in den EGVertrag. Die Mitgliedsstaaten hatten schon seit 1994 die Möglichkeit diskutiert, in der Beschäftigungspolitik zusammenzuarbeiten. Auf dem Essener Gipfel hatten sie die freiwillige Koordinierung nationaler Beschäftigungspolitik durch Zielvereinbarungen, Benchmarking und wechselseitige Überwachung beschlossen. ${ }^{16} \mathrm{Im}$ Vorfeld von Amsterdam herrschte Streit um die Aufnahme eines eigenen Beschäftigungstitels in den Vertrag. Die Regierungen von Deutschland, Frankreich und Großbritannien lehnten dies ab, weil sie höhere Ausgaben und eine übereifrige Kommission fürchteten. Nach den Wahlen in Frankreich und Großbritannien war allerdings die

14 Frankreich erlebte zum dritten Mal eine Phase der Kohabitation. Jacques Chirac war Präsident und Lionel Jospin der neu gewählte Regierungschef.

15 Damit bestätigte sich Piersons (1996: 155) Vorhersage, dass ein einziger Labour-Sieg das britische Opt-out beenden würde.

16 Hix und Lord (1997: 194) argumentieren, dass unter der Regie der christlich-liberalen Koalition in Deutschland das unverbindliche Essener Verfahren beschlossen wurde, um weiter gehende Vorschläge sozialdemokratischer Regierungen abzuwehren. 
Regierung Kohl in ihrer Ablehnung isoliert. Vor allem Jospin machte seine Zustimmung zum Stabilitäts- und Wachstumspakt (SWP) vom Schicksal des Beschäftigungstitels abhängig. Zu guter Letzt wurde sowohl der SWP verabschiedet als auch der Beschäftigungstitel aufgenommen. Der parteipolitische Kompromiss bestand darin, ihn zwar aufzunehmen, aber von einem Kompetenztransfer oder Sanktionsmöglichkeiten abzusehen.

Der neu eingeführte Titel VIII legte »Förderung der Beschäftigung als Angelegenheit von gemeinsamem Interesse« (Art. 126/1, EGV) fest. Gleichzeitig unterstrich Artikel 127, dass die Zusammenarbeit gefördert, aber die Zuständigkeit der Mitgliedsstaaten beachtet werden sollte. Für die Europäische Beschäftigungsstrategie wurde folgendes Verfahren vereinbart:

- Der Europäische Rat prüft die Beschäftigungslage in der Gemeinschaft und nimmt hierzu Schlussfolgerungen an;

- der Rat beschließt aufbauend auf den Vorgaben des Europäischen Rats beschäftigungspolitische Leitlinien;

- die Regierungen entwerfen Nationale Aktionspläne (NAP), die Maßnahmen zur Verwirklichung der Leitlinien auflisten;

- Kommission und Rat verabschieden den Gemeinsamen Beschäftigungsbericht, der die Politik der Mitgliedsstaaten bewertet und Empfehlungen ausspricht.

Während dieses Verfahren Teil des Vertrags ist (Art. 128), kann die Umsetzung der Leitlinien nicht erzwungen werden. Die Regierungen konnten sich in der Beschäftigungspolitik nicht auf verbindliche Ziele verständigen, wie dies bei Schulden, Haushaltsdefiziten und der Inflationsrate gelungen war. Alle Versuche der Kommission, ambitioniertere beschäftigungspolitische Leitlinien und quantifizierbare Ziele zu definieren, scheiterten an der politischen Uneinigkeit regierender Mitte-linksParteien (Pollack 2000: 269). Dafür gibt es zwei Gründe. Abbildungen 2 und 3 zeigen zum einen, dass mit der Linksverschiebung des Gravitationszentrums eine abnehmende Unterstützung für die europäische Integration einherging. Die politische Grundlage für weitere Kompetenztransfers war nach Maastricht erschöpft. Zum anderen verdeutlicht Abbildung 6 die veränderte ideologische Ausrichtung sozialdemokratischer Parteien seit den siebziger Jahren. Nicht nur New Labour, sondern die Sozialdemokratie insgesamt hatte sich am Ende des zwanzigsten Jahrhunderts vom Interventionismus verabschiedet. In deutlichem Gegensatz zu den siebziger Jahren konvergierten die untersuchten Parteien hinsichtlich ihrer geringen Zustimmung zur Regulierung der Märkte (Abb. 7). Und während die Sozialdemokratie noch immer den Wohlfahrtsstaat grundsätzlich begrüßte, begann sie, dessen Aufgaben neu zu definieren. Aktivierung und Beschäftigungsfähigkeit rückten nun stärker in den Vordergrund. Im Ergebnis bedeuteten diese Veränderungen, dass der Dritte Weg mit der Sozialdemokratie der siebziger Jahre wenig gemeinsam hatte. ${ }^{17}$ Anstatt ein Gegenkonzept zur Währungsunion zu formulieren, wollten diese Parteien sie sozial- und arbeitsmarktpolitisch ergänzen.

17 Darüber hinaus wachten die Sozialdemokraten darüber, ihre Kompetenzen in der Sozialund Arbeitsmarktpolitik nicht an Europa zu verlieren, um in diesen Feldern weiterhin bei Wahlen punkten zu können (siehe Ladrech 2003: 119). 
Philip Manow/Armin Schäfer/Hendrik Zorn: Europäische Sozialpolitik in den Jahren 1957-2003

Abbildung 6: Links-rechts-Index nach Laver/Hunt (1992)

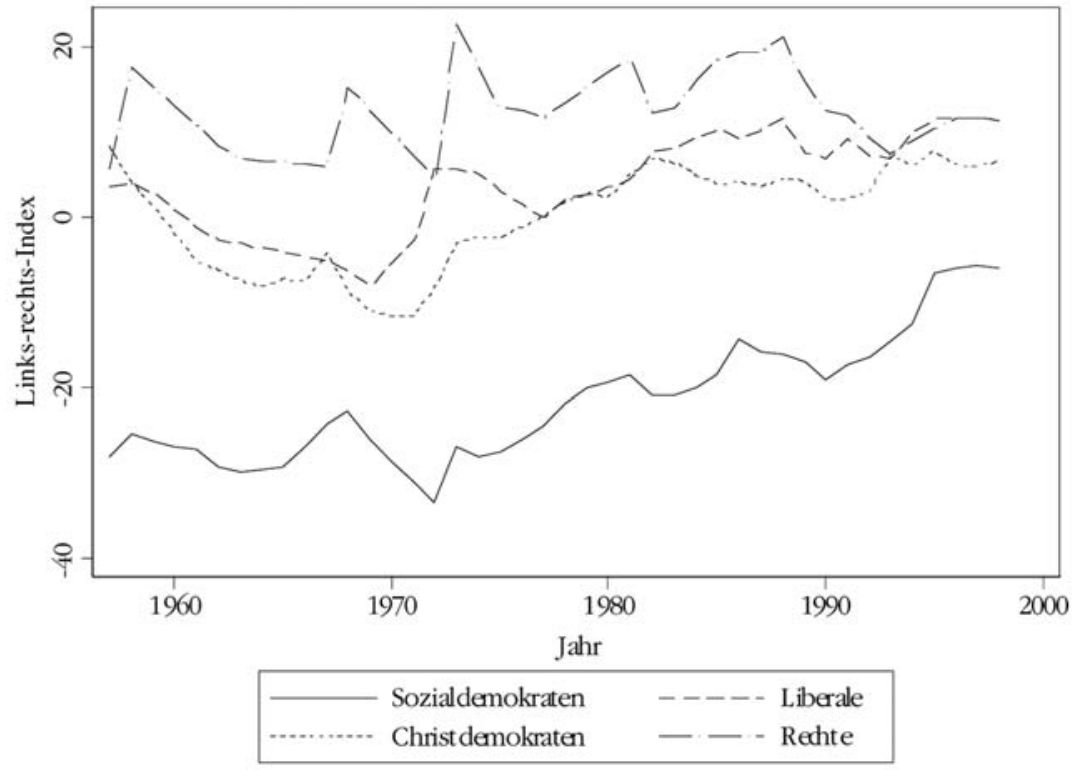

Abbildung 7 Befürwortung staatlicher Eingriffe in das Marktgeschehen

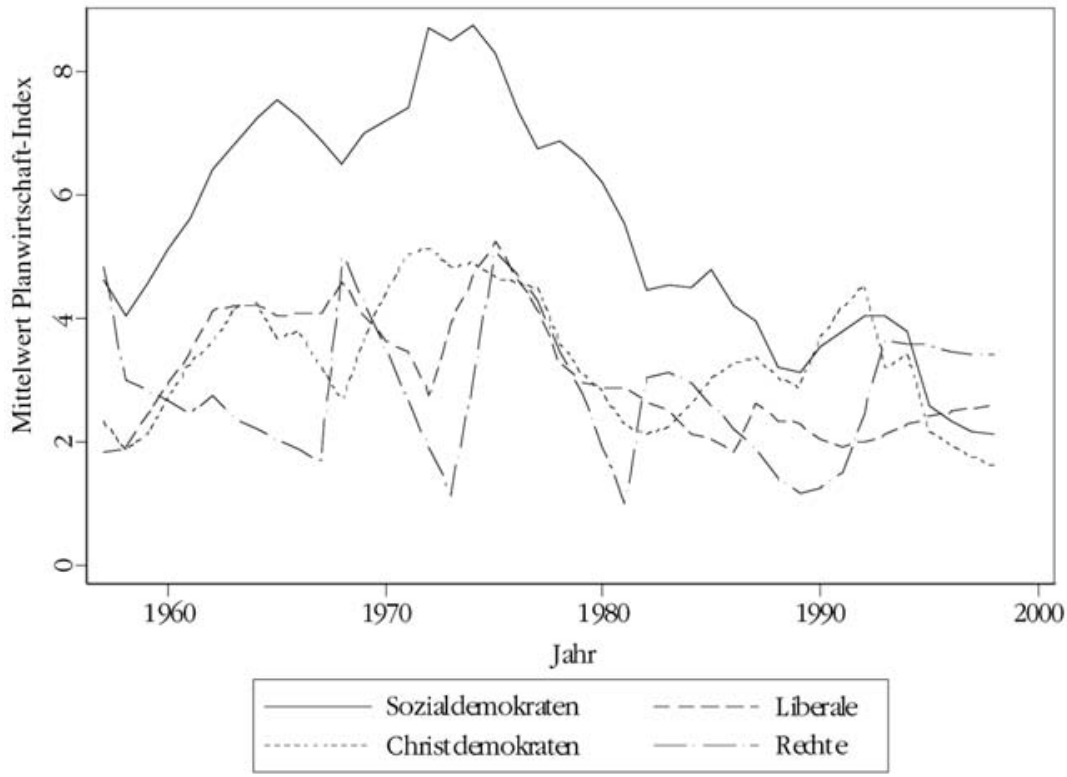


Der Lissabonner Gipfel im März 2000 war die Geburtsstunde der OMC. In elf der 15 Mitgliedsstaaten regierten Mitte-links-Koalitionen. Sie beschlossen ein strategisches Ziel für die EU: Sie solle bis 2010 »der wettbewerbsfähigste und dynamischste wissensbasierte Wirtschaftsraum der Welt werden«, fähig zu nachhaltigem Wachstum, Vollbeschäftigung und größerem sozialen Zusammenhalt (Europäischer Rat 2000). Diese Ziele wollte man nicht durch zusätzliche gesetzliche Regeln verwirklichen, sondern - wie in der Beschäftigungsstrategie erprobt - durch die Koordinierung nationaler Politiken. Inzwischen ist diese Koordinierungsmethode auf mehr als zehn weitere Bereiche ausgeweitet worden, z. B. auf die Renten- und Armutspolitik (Social Inclusion). Die OMC ist besonders dann attraktiv, wenn keine vertragliche Grundlage besteht oder hergestellt werden kann. Während somit neue Bereiche der Sozialpolitik erfasst werden, bleiben die getroffenen Vereinbarungen rechtlich unverbindlich (Schäfer 2005).

Über die letzten Jahre sind Ziele und Aufgaben der EU Gegenstand parteipolitischer Auseinandersetzung geworden. Liesbeth Hooghe, Gary Marks und Carole Wilson (2004: 127-128) zeigen, dass linke und rechte Parteien beispielsweise die Notwendigkeit von Markteingriffen gegensätzlich beurteilen. Parteien links von der Mitte befürworten prinzipiell eine Umwelt-, Kohäsions- und Beschäftigungspolitik der EU, Parteien rechts von der Mitte lehnen dies ab. Die OMC war der sozialdemokratische Versuch, neue Felder der Sozialpolitik der Zusammenarbeit zuzuführen, ohne jedoch die bestehende Wirtschaftsverfasssung herauszufordern. Sie setzten dabei auf das Prinzip der Freiwilligkeit.

Am Ende dieses Abschnitts folgen zwei Schlussfolgerungen: Erstens, die Heterogenität des Rats erscheint heute als Garant des ordoliberalen Ideals einer Trennung von europäischer Wirtschafts- und nationaler Sozialordnung, weil Kompromisse zwischen den unterschiedlichen im Ministerrat vertretenen politischen Zielen schwieriger werden. Zweitens, während die parteipolitische Kontroverse über die angemessenen EU-Kompetenzen zunimmt, verringert sich gleichzeitig der vorhandene Entscheidungsspielraum. Die sozialdemokratischen Regierungen in der zweiten Hälfte der neunziger Jahre waren an die Institutionen gebunden, die ihre - häufig: christdemokratischen - Vorgänger geschaffen hatten.

\section{Schluss}

In diesem Aufsatz haben wir die politische Zusammensetzung intergouvernementaler EU-Institutionen untersucht und exemplarisch für die europäische Sozialpolitik argumentiert, dass sich Veränderungen in Europas parteipolitischem Gravitationszentrum auf den Modus der europäischen Integration auswirken. Änderungen in der politischen Position von EU-Mitgliedsregierungen wirken sich auf einzelne Felder der Europapolitik aus, aber auch generell auf die Integrationsfreundlichkeit. Die erhebliche Variation in diesen Werten macht die aggregierte Regierungszusammensetzung $\mathrm{zu}$ einer fruchtbaren erklärenden Variable. Aus unseren Ergebnissen schließen wir, dass die ideologische Ausrichtung von Regierungen wichtig für die 
Definition des nationalen Interesses ist. Nicht monolithische Staaten verhandeln international miteinander, sondern Regierungen, deren politische Glaubwürdigkeit auch davon abhängt, dass sie international keine völlig andere Politik als »zuhause« vertreten. In diesem Zusammenhang erscheint der Nachweis zyklischer Schwankungen des politischen Gravitationszentrums, den unsere empirische Analyse erbrachte, bedeutsam. Der Einfluss von Änderungen in der parteipolitischen Zusammensetzung von Regierungen auf die »Mehrheiten « in internationalen Gremien ist nicht stochastisch; stattdessen gibt es länderübergreifende Trends. Ließe sich ein solcher Zusammenhang auch innerhalb einer größeren Staatengruppe nachweisen, wäre es plausibel, ebenso einen parteipolitischen Einfluss auf die Arbeit anderer internationaler Organisationen anzunehmen. So haben sich beispielsweise die arbeitsmarktpolitischen Empfehlungen von OECD und EU Ende der neunziger Jahre unter der Dominanz sozialdemokratischer Regierungen deutlich angenähert. Zukünftige Forschung könnte etwa der Frage nachgehen, ob sich der Grad parteipolitischer Homogenität auch auf Einigungschancen und Inhalte der G-8 Gipfeltreffen ausgewirkt hat.

In den Daten zur Europäischen Union lassen sich einige bemerkenswerte Trends erkennen. In den fünfziger und sechziger Jahren dominierten in den Mitgliedsstaaten Mitte-rechts-, vor allem aber christdemokratische Parteien. Erst in den Siebzigern gab es eine erste Welle sozialdemokratisch geführter Regierungen, die jedoch in den Achtzigern mit den Wahlerfolgen konservativ-liberaler Parteien endete. Die Rückkehr der Sozialdemokratie an die Macht erfolgte erst wieder in der zweiten Hälfte der neunziger Jahre - in einem bis dahin nicht gekannten Ausmaß. Ein zweiter Befund ist, dass die Heterogenität des Rats mit jeder Erweiterungsrunde zugenommen hat und bisherige Erweiterungen das Gravitationszentrum jeweils nach links verschoben haben. ${ }^{18}$ Als Drittes lässt sich der Wechsel von beschleunigter Integration zu Phasen größerer Integrationsskepsis hervorheben. Insbesondere ging mit der Linksverschiebung des Gravitationszentrums ein deutlicher Rückgang der Unterstützung für die Integration einher. Dieses Ergebnis bestätigt die Vermutung, dass Parteien links von der Mitte eine größere Distanz zur EU aufweisen. Die Parteifamilien unterscheiden sich systematisch in der Beurteilung der europäischen Integration. Christdemokratische Parteien halten die EU für wichtiger und unterstützen sie stärker als konservative oder sozialdemokratische Parteien. Schließlich finden sich zwischen den Parteien deutliche Unterschiede hinsichtlich ihrer Einstellungen zum Wohlfahrtsstaat, zu Markteingriffen oder dem freien Spiel der Marktkräfte. Auch hier beobachten wir eine erhebliche Variation über Zeit. Für den betrachteten Zeitraum lässt sich eine ideologische Rechtsverschiebung sowohl von Mitte-rechtsals auch Mitte-links-Parteien konstatieren. Das heißt, die sozialdemokratischen Regierungen waren in der zweiten Hälfte der neunziger Jahre deutlich weniger interventionistisch orientiert als in der Vergangenheit. Sie hatten mittlerweile die liberale Wirtschaftsverfassung der EG weitgehend akzeptiert.

Die Rekonstruktion der europäischen Sozialpolitik seit den Römischen Verträgen diente uns in diesem Aufsatz als exemplarische Fallstudie. Wir argumentieren, dass

18 Die Osterweiterung wird von unseren Daten nicht abgedeckt. 
es auch eine parteipolitische Erklärung dafür gibt, weshalb in Europa die soziale Dimension der wirtschaftlichen Integration untergeordnet blieb. Wichtig in diesem Zusammenhang erscheint uns die frühe Weichenstellung im EWG-Vertrag, in diesem Bereich nur begrenzt Souveränität abzugeben. In diesem Punkt bestand zwischen linken und rechten Parteien ein unausgesprochener Konsens. Im Ergebnis entstand mit den Römischen Verträgen eine Wirtschaftsverfassung, die den Wünschen deutscher Ordoliberaler entgegen kam: Das Recht verpflichtete die Gemeinschaftsinstitutionen auf den liberalen Markt, während die Selbstblockade der Politik eine Harmonisierung nationaler Sozialpolitik oder gar deren Europäisierung verhinderte. In der Folge bewegte sich die Integration entlang dieses Pfads, bis Mitte-rechtsRegierungen in der zweiten Hälfte der achtziger Jahre sie als externen Hebel zur Beschneidung des nationalen Wohlfahrtsstaats entdeckten. Im Gegensatz dazu wollte die Sozialdemokratie die Währungsunion mit dem Europäischen Sozialmodell versöhnen und führte die Offene Methode der Koordinierung in der Beschäftigungs- und Sozialpolitik ein.

Als generelle und empirisch überprüfbare Thesen lassen sich aus der bisherigen Argumentation die folgenden Punkte gewinnen: Erstens werden nationale Interessen durch die Präferenzen regierender Parteien gefiltert. Zweitens beeinflusst die parteipolitische Zusammensetzung der Mitgliedschaft eines internationalen Regimes Erfolgswahrscheinlichkeit und Inhalte der Kooperation. Drittens erleichtert parteipolitische Heterogenität es supranationalen Akteuren, delegierte Entscheidungsbefugnisse in ihrem eigenen Interesse zu nutzen, da sie die Auftraggeber gegeneinander ausspielen können. Schließlich - und viertens - wirken sich die politischen Präferenzen der Regierung auf die Umsetzung und Befolgung internationaler Vereinbarungen aus. Natürlich ist die Parteipolitik nicht der einzige erklärungsrelevante Faktor. Gemessen an seiner Erklärungsrelevanz wurde diesem Faktor unserem Eindruck nach aber bisher sowohl in der Europaforschung als auch generell in den Internationalen Beziehungen zu wenig Aufmerksamkeit zuteil.

\section{Anhang}

Die Experten-Einstufungen zur Messung der Links-rechts-Positionen stammen aus der Umfrage von Laver/Hunt (1992). Wir beziehen uns auf die »increase services vs. cut taxes «- Dimension. Für Parteien, die in diesem survey nicht eingestuft wurden, haben wir andere Experten-Einstufungen (wie z. B. Katz/Mair) herangezogen und linear transformiert, um sie der Laver/Hunt-Skala anzupassen. Für Parteien, die in keinem survey erfasst wurden, haben wir die entsprechende Position über den Linksrechts-Index des Comparative Manifesto Project ermittelt. Alle Beobachtungen für eine Partei wurden dabei gemittelt (um Ausreißer zu glätten) und ebenfalls linear transformiert.

Die europäischen Center of Gravity (CoG)-Werte wurden in zwei Schritten berechnet. Zunächst haben wir die jährlichen länderspezifischen CoG ermittelt. Bei Koalitionsregierungen wurden die Links-rechts-Positionen mit dem Anteil an 
Regierungssitzen gewichtet. Traten Regierungswechsel auf, gingen die individuellen Werte mit dem Gewicht der Regierungsdauer (in Tagen) ein. Die europäischen CoG-Werte wurden dann in einem zweiten Schritt als arithmetisches Mittel der Länder-Werte berechnet. Der Wert für Italien im Jahr 1995 wurde nicht berücksichtigt, da dort fast ausschließlich eine Technokratenregierung amtierte.

Um die Daten des Comparative Manifesto Project für unsere Analysen verwenden zu können, mussten wir einige Anpassungen vornehmen. So wurden Parteien, für die keine Auswertung vorgenommen wurde, aus der Analyse ausgeklammert. Für die Wahlen, die noch nicht im Projekt erfasst wurden, haben wir die Werte der jüngsten erfassten Wahlen verwendet. Selbstverständlich haben wir die Analyse von Präferenzverschiebungen auf die Fälle begrenzt, für die Daten vorhanden waren.

Als Quellen für die Zusammensetzung von Regierungen dienten die folgenden Dokumente: Keesing's Record of World Events; Datenhandbücher des European Journal of Political Research (Woldendorp/Keman/Budge 2000); Mackie/Rose (1991); verschiedene länderspezifische Quellen.

\section{Literatur}

Abelshauser, Werner 1996: Erhard oder Bismarck? Die Richtungsentscheidung der deutschen Sozialpolitik am Beispiel der Reform der Sozialversicherung, in: Geschichte und Gesellschaft 22: 3, 375-391.

Alter, Karen J. 1998: Who Are the »Masters of the Treaty«? European Governments and the European Court of Justice, in: International Organization 52: 1, 121-147.

Alter, Karen J./Meunier-Aitsahalia, Sophie 1994: Judicial Politics in the European Community: European Integration and the Pathbreaking Cassis de Dijon Decision, in: Comparative Political Studies 26: 4, 535-561.

Alter, Karen J./Vargas, Jeannette 2000: Explaining Variation in the Use of European Litigation Strategies: European Community Law and British Gender Equality Policy, in: Comparative Political Studies 33: 4, 452-482.

Aspinwall, Mark, 2002: Preferring Europe: Ideology and National Preferences on European Integration, in: European Union Politics 3: 1, 81-111.

Bartolini, Stefano 2005: Restructuring Europe: Centre Formation, System Building and Political Structuring between the Nation-State and the European Union, Oxford.

Budge, Ian/Klingemann, Hans-Dieter/Bara, Judith/Tanenbaum, Eric/Volkens, Andrea (Hrsg.) 2001: Mapping Policy Preferences: Estimates for Parties, Electors, and Governments 1945-1998, Oxford.

Burley, Anne-Marie/Mattli, Walter 1993: Europe before the Court: A Political Theory of Legal Integration, in: International Organization 47: 1, 41-76.

Caporaso, James A. 1992: International Relations Theory and Multilateralism: The Search for Foundations, in: International Organization 46: 3, 599-632.

Carstens, Karl 1976: Das Eingreifens Adenauers in die Europa-Verhandlungen im November 1956, in: Blumenwitz, Dieter (Hrsg.): Konrad Adenauer und seine Zeit, Stuttgart, 591602.

Castles, Francis C./Mair, Peter 1984: Left-Right Political Scales: Some Expert Judgements, in: European Journal of Political Research 12: 1, 73-88. 
Dyson, Kenneth/Featherstone, Kevin 1999: The Road to Maastricht: Negotiating Economic and Monetary Union, Oxford.

Ehlermann, Claus-Dieter 1995: Comment on Manfred E. Streit and Werner Mussler: »The Economic Constitution of the European Community: From >Rome < to >Maastricht « $\ll$, in: European Law Journal 1: 1, 84-85.

Europäischer Rat 2000: Schlussfolgerungen des Vorsitzes (Lissabon, 23.-24.3.2000), in: http://www.europarl.eu.int/summits/lis1_de.htm; 26.3.2006.

Falkner, Gerda 1998: EU Social Policy in the 1990s: Towards a Corporatist Policy Community, London.

Genschel, Philipp 1998: Markt und Staat in Europa, in: Politische Vierteljahresschrift 39: 1, 55-79.

Gerber, David J. 1994: Constitutionalizing the Economy: German Neo-Liberalism, Competition Law and the »New« Europe, in: American Journal of Comparative Law 42: 1, 2584.

Green Cowles, Maria 1995: Setting the Agenda for a New Europe: The ERT and EC 1992, in: Journal of Common Market Studies 33: 4, 501-526.

Gross, Donald A./Sigelman, Lee 1984: Comparing Party Systems: A Multidemensional Approach, in: Comparative Politics 16: 4, 463-479.

Hall, Peter A. 1999: The Political Economy of Europe in an Era of Interdependence, in: Kitschelt, Herbert/Lange, Peter/Marks, Gary/Stephens, John D. (Hrsg.): Continuity and Change in Contemporary Capitalism, Cambridge, 135-163.

Hentschel, Volker 1998: Ludwig Erhard. Ein Politikerleben, Berlin.

Héritier, Adrienne 1996: The Accommodation of Diversity in European Policy-Making and Its Outcomes: Regulatory Policy as a Patchwork, in: Journal of European Public Policy 3: 2, 149-167.

Hinich, Melvin J./Munger, Michael C. 1992: A Spatial Theory of Ideology, in: Journal of Theoretical Politics 4: 1, 5-30.

Hix, Simon 1999: The Political System of the European Union, New York, NY.

Hix, Simon/Lord, Christopher 1997: Political Parties in the European Union, Houndmills, NY.

Hooghe, Liesbeth/Marks, Gary/Wilson, Carole J. 2004: Does Left/Right Structure Party Positions on European Integration?, in: Marks, Gary/Steenbergen, Marco R. (Hrsg.): European Integration and Political Conflict, Cambridge, 120-140.

Höpner, Martin 2005: Parteien oder Nationen? Die zwei Konfliktlinien der europäischen Finanzmarktintegration, in: Zeitschrift für Internationale Beziehungen 12: 2, 251-273.

Huber, John/Inglehart, Ronald 1995: Expert Interpretations of Party Space and Party Locations in 42 Societies, in: Party Politics 1: 1, 73-111.

Joerges, Christian 2003: Recht, Wirtschaft und Politik im Prozess der Konstitutionalisierung Europas, in: Jachtenfuchs, Markus/Kohler-Koch, Beate (Hrsg.): Europäische Integration, Opladen, 183-218.

Johansson, Karl Magnus 2002: Another Road to Maastricht: The Christian Democrat Coalition and the Quest for European Union, in: Journal of Common Market Studies 40: 5, 871-893.

Kalyvas, Stathis N. 1996: The Rise of Christian Democracy in Europe, Ithaca, NY.

Keohane, Robert O. 1984: After Hegemony: Cooperation and Discord in the World Political Economy, Princeton, NY.

Keohane, Robert O./Hoffmann, Stanley 1990: Conclusions: Community Politics and Institutional Change, in: Wallace, William (Hrsg.): The Dynamics of European Integration, London, 276-300.

Kowalsky, Wolfgang 1999: Europäische Sozialpolitik. Ausgangsbedingungen, Antriebskräfte und Entwicklungspotentiale, Opladen. 
Küsters, Hanns Jürgen 1982: Die Gründung der Europäischen Wirtschaftsgemeinschaft, Baden-Baden.

Küsters, Hanns Jürgen 1989: The Treaties of Rome (1955-57), in: Pryce, Roy (Hrsg.): The Dynamics of European Union, London, 78-104.

Ladrech, Robert 2003: The Left and the European Union, in: Parliamentary Affairs 56: 4, 112124.

Lange, Peter 1993: Maastricht and the Social Protocol: Why Did They Do It?, in: Politics and Society 21: 1, 5-36.

Laver, Michael/Hunt, Ben W. 1992: Policy and Party Competition, New York, NY.

Lee, Sabine 1995: German Decision-Making Elites and European Integration: German »Europapolitik « during the Years of the EEC and Free Trade Negotiations, in: Deighton, Anne (Hrsg.): Building Postwar Europe, Basingstoke, 38-54.

Leibfried, Stephan/Pierson, Paul 2000: Social Policy: Left to Courts and Markets?, in: Wallace, Helen/Wallace, William (Hrsg.): Policy-Making in the European Union, Oxford, 267-292.

Lynch, France M. B. 1997: France and the International Economy: From Vichy to the Treaty of Rome, London.

Lynch, Frances M. B. 1994: Restoring France: The Road to Integration, in: Milward, Alan S./ Lynch, Frances M. B./Ranieri, Ruggero/Romero, Federico/Sørensen, Vibeke (Hrsg.): The Frontier of National Sovereignty: History and Theory 1945-1992, London, 59-87.

Mackie, Thomas T./Rose, Richard 1991: The International Almanac of Electoral History, 3. Auflage, Washington, DC.

Mair, Peter 2001: The Limited Impact of Europe on National Party Systems, in: Goetz, Klaus H./ Hix, Simon (Hrsg.): Europeanised Politics? European Integration and National Political System, London, 27-51.

Manow, Philip 1999: Reziproker Interessenausgleich in transnationalen Verhandlungssystemen und in Konkordanzdemokratien, in: Schweizerische Zeitschrift für Politische Wissenschaft 5: 2, 5-37.

Marks, Gary/Wilson, Carole J./Ray, Leonard 2002: National Political Parties and European Integration, in: American Journal of Political Science 46: 3, 585-594.

Mattila, Mikko 2004: Contested Decisions: Empirical Analysis of Voting in the EU Council of Ministers, in: European Journal of Political Research 43: 1, 29-50.

Mestmäcker, Ernst-Joachim 2003: Wirtschaft und Verfassung in der Europäischen Union. Beiträge zu Recht, Theorie und Politik der europäischen Integration, Baden-Baden.

Milward, Alan S. 2000: The European Rescue of the Nation-State, London.

Moravcsik, Andrew 1991: Negotiating the Single European Act, in: Keohane, Robert O./Hoffmann, Stanley (Hrsg.): The New European Community: Decisionmaking and Institutional Change, Boulder, CO, 41-84.

Moravcsik, Andrew 1993: Preferences and Power in the European Community: A Liberal Intergouvernmentalist Approach, in: Journal of Common Market Studies 31: 4, 471-524.

Moravcsik, Andrew 1997: Warum die Europäische Union die Exekutive stärkt: Innenpolitik und internationale Kooperation, in: Wolf, Klaus-Dieter (Hrsg.): Projekt Europa im Übergang? Probleme, Modelle und Strategien des Regierens in der Europäischen Union, Baden-Baden, 211-269.

Moravcsik, Andrew 1998: The Choice for Europe: Social Purpose and State Power from Messina to Maastricht, London.

Müller-Armack, Alfred 1966: Die Wirtschaftsordnung des Gemeinsamen Marktes, in: MüllerArmack, Alfred (Hrsg.): Wirtschaftsordnung und Wirtschaftspolitik. Studien und Konzepte zur sozialen Marktwirtschaft und zur europäischen Integration, Freiburg, 401-415. 
Ophüls, Carl Friedrich 1961/62: Gründzüge europäischer Wirtschaftsverfassung, in: Zeitschrift für das gesamte Handels- und Wirtschaftsrecht 124, 136-181.

Pierson, Paul 1996: The Path to European Integration: A Historical Institutionalist Analysis, in: Comparative Political Studies 29: 2, 123-163.

Pollack, Mark A. 1997: Delegation, Agency, and Agenda Setting in the European Community, in: International Organization 51: 1, 99-134.

Pollack, Mark A. 2000: A Blairite Treaty: Neo-Liberalism and Regulated Capitalism in the Treaty of Amsterdam, in: Neunreither, Karlheinz/Wiener, Antje (Hrsg.): European Integration after Amsterdam: Institutional Dynamics and Prospects for Democracy, Oxford, 266-289.

Pollack, Mark A. 2003: The Engines of European Integration: Delegation, Agency, and Agenda Setting in the EU, Oxford.

Putnam, Robert 1988: Democracy and Domestic Politics: The Logic of Two-Level Games, in: International Organization 42: 3, 427-460.

Ray, Leonard 1999: Measuring Party Orientation towards European Integration: Results from an Expert Survey, in: European Journal of Political Research 36: 6, 283-306.

Rhodes, Martin 1998: Das Verwirrspiel der »Regulierung«. Industrielle Beziehungen und »soziale Dimension«, in: Leibfried, Stephan/Paul, Pierson (Hrsg.): Standort Europa. Sozialpolitik zwischen Nationalstaat und Europäischer Integration, Frankfurt a. M., 100154.

Reif, Karlheinz/Schmitt, Hermann 1980: Nine Second-Order National Elections: A Conceptual Framework for the Analysis of European Election Results, in: European Journal of Political Research 8: 1, 3-45.

Reif, Karlheinz 1984: National Electoral Cycles and European Elections 1979 and 1984, in: Electoral Studies 3: 3, 244-255.

Ross, George 1995: Jacques Delors and European Integration, Cambridge.

Sandholtz, Wayne/Stone Sweet, Alec (Hrsg.) 1998: European Integration and Supranational Governance, Oxford.

Sandholtz, Wayne/Zysman, John 1989: 1992: Recasting the European Bargain, in: World Politics 42: 1, 95-128.

Schäfer, Armin 2005: Die neue Unverbindlichkeit. Wirtschaftspolitische Koordinierung in Europa, Frankfurt a. M.

Scharpf, Fritz W. 1999: Regieren in Europa. Effektiv und demokratisch?, Frankfurt a. M.

Scharpf, Fritz W. 2002: The European Social Model: Coping with the Challenges of Diversity, in: Journal of Common Market Studies 40: 4, 645-670.

Schmidt, Manfred G. 1996: When Parties Matter: A Review of the Possibilities and Limits of Partisan Influence on Public Policy, in: European Journal of Political Research 30: 2, 155-183.

Schmidt, Manfred G. 2005: Sozialpolitik in Deutschland. Historische Entwicklung und internationaler Vergleich, 3. Auflage, Wiesbaden.

Stone Sweet, Alec/Sandholtz, Wayne/Fligstein, Neil (Hrsg.) 2002: The Institutionalization of Europe, Oxford.

Stone Sweet, Alec 2004: The Judicial Construction of Europe, Oxford.

Streeck, Wolfgang 1998: Vom Binnenmarkt zum Bundesstaat? Überlegungen zur politischen Ökonomie der europäischen Sozialpolitik, in: Leibfried; Stephan/Pierson, Paul (Hrsg.): Standort Europa. Sozialpolitik zwischen Nationalstaat und Europäischer Integration. Frankfurt a. M., 369-421.

Streit, Manfred E./Mussler, Werner 1995: The Economic Constitution of the European Community: From »Rome« to »Maastricht«, in: European Law Journal 1: 1, 5-30. 
Treib, Oliver 2003: Die Umsetzung von EU-Richtlinien im Zeichen der Parteipolitik: eine akteurszentrierte Antwort auf die Misfit-These, in: Politische Vierteljahresschrift 44: 4, 506-528.

Tsebelis, George 1994: The Power of the European Parliament as a Conditional Agenda Setter, in: American Political Science Review 88: 1, 128-141.

Tsebelis, George/Kreppel, Amie 1998: The History of Conditional Agenda-Setting in European Institutions, in: European Journal of Political Research 33: 1, 41-71.

Tsoukalis, Loukas 1993: The New European Economy: The Politics and Economics of Integration, 2. Auflage, Oxford.

van der Eijk, Cees/Franklin, Mark N. 1996: Choosing Europe? The European Electorate and National Politics in the Face of the Union, Ann Arbor, MI.

van der Eijk, Cees/Franklin, Mark N./Marsh, Michael 1996: What Voters Teach Us about Europe-Wide Elections: What Europe-Wide Elections Teach Us about Voters, in: Electoral Studies 15: 2, 149-166.

von der Groeben, Hans 1987: Der Einfluß liberaler Vorstellungen auf die Gründungsverträge der Europäischen Gemeinschaften, in: von der Groeben, Hans: Die Europäische Gemeinschaft und die Herausforderungen unserer Zeit. Aufsätze und Reden 1967-1987, Baden-Baden, 183-199.

Woldendorp, Jaap/Keman, Hans/Budge, Ian 2000: Party Government in 48 Democracies (1945-1998): Composition - Duration - Personnel, Dordrecht.

Wolf, Dieter/Zangl, Bernhard 1996: The European Economic and Monetary Union: »TwoLevel Games « and the Formation of International Institutions, in: European Journal of International Relations 2: 3, 355-393.

Woolley, John T. 1994: Linking Political and Monetary Union: The Maastricht Agenda and German Domestic Politics, in: Eichengreen, Barry J./Frieden, Jeffry (Hrsg.): The Political Economy of European Monetary Unification, Boulder, CO, 67-86.

Zürn, Michael 1992: Interessen und Institutionen in der internationalen Politik. Grundlegung und Anwendungen des situationsstrukturellen Ansatzes, Opladen. 